\title{
Role of Post-translational Modifications in Influenza A Virus Life Cycle and Host Innate Immune Response
}

\author{
Jiao Hu ${ }^{1,2,3}$, Lei Zhang ${ }^{1,2,3}$ and Xiufan Liu ${ }^{1,2,3 *}$ \\ ${ }^{1}$ Animal Infectious Disease Laboratory, School of Veterinary Medicine, Yangzhou University, Yangzhou, China, ${ }^{2}$ Jiangsu \\ Co-innovation Center for Prevention and Control of Important Animal Infectious Diseases and Zoonosis, Yangzhou University, \\ Yangzhou, China, ${ }^{3}$ Key Laboratory of Prevention and Control of Biological Hazard Factors (Animal Origin) for Agrifood Safety \\ and Quality, Ministry of Agriculture of China, Yangzhou University, Yangzhou, China
}

OPEN ACCESS

Edited by:

Romain Volmer,

Ecole Nationale Vétérinaire

de Toulouse, France

Reviewed by:

Olivier Terrier,

UMR 5308 Centre International de Recherche en Infectiologie (CIRI),

France

Linda Brunotte

University of Münster, Germany

*Correspondence:

Xiufan Liu

xfliu@yzu.edu.cn

Specialty section:

This article was submitted to

Virology,

a section of the journa

Frontiers in Microbiology

Received: 04 December 2019

Accepted: 14 August 2020

Published: 04 September 2020

Citation:

Hu J, Zhang L and Liu X (2020)

Role of Post-translational

Modifications in Influenza A Virus Life

Cycle and Host Innate Immune

Response.

Front. Microbiol. 11:517461.

doi: $10.3389 /$ fmicb.2020.517461
Throughout various stages of its life cycle, influenza A virus relies heavily on host cellular machinery, including the post-translational modifications (PTMs) system. During infection, influenza virus interacts extensively with the cellular PTMs system to aid in its successful infection and dissemination. The complex interplay between viruses and the PTMs system induces global changes in PTMs of the host proteome as well as modifications of specific host or viral proteins. The most common PTMs include phosphorylation, ubiquitination, SUMOylation, acetylation, methylation, NEDDylation, and glycosylation. Many PTMs directly support influenza virus infection, whereas others contribute to modulating antiviral responses. In this review, we describe current knowledge regarding the role of PTMs in different stages of the influenza virus replication cycle. We also discuss the concerted role of PTMs in antagonizing host antiviral responses, with an emphasis on their impact on viral pathogenicity and host range.

Keywords: influenza virus, post-translational modifications, replication cycle, pathogenesis, virus-host interaction, innate immune response

Abbreviations: ANP32A, acidic nuclear phosphoprotein 32 family member A; CARD, caspase recruitment domains; CDC25B, cell division cycle 25 B; CDKs, cyclin dependent kinases; CK1 $\alpha$, casein kinase 1; CNOT4, Ccr4-not transcription complex subunit 4; CRM1, chromosome region maintenance 1 protein homolog; DNMT, DNA methyltransferase; DNMT3B, DNA methyltransferase; GCN5, general control of amino-acid synthesis 5-like 2; HA, hemagglutinin; HDAC1, histone deacetylase 1; HDAC6, histone deacetylase 6; HDAC6/8, histone deacetylases6/8; HIV, human immunodefciency virus type-1; HSP90, heat shock protein 90; IAV, influenza A viruses; IFITM3, IFN-induced transmembrane protein 3; IFITM3, interferoninduced transmembrane protein 3; IFN, interferon; IFNAR1, type I IFN receptor 1; IRF3, interferon regulatory factor 3; M, matrix protein; MAVS, mitochondrial antiviral-signaling protein; MLC, myosin light chain; NA, neuraminidase; NEMO, NF-Kappa-B essential modulator; NES2, nuclear export signal 2; NP, nucleoprotein; NS, non-structural protein; OAS, oligo (A) synthetase; PA, polymerase acidic protein; PB1, polymerase basic protein 1; PB2, polymerase basic protein 2; PCAF, P300/CBP-associated factor; PKC $\alpha$, protein kinase C $\alpha$; PKM, pyruvate kinase M; PKR, protein kinase R; PTMs, posttranslational modifications; RanBP3, ran-binding protein 3; RdRP, RNA-dependent RNA polymerase RdRp complex; RIG-I, retinoic acid-induced gene 1 protein; SIM-like, SUMO interaction motif-like; TRAF3, TNF receptor-associated factor 3; TRIM, tripartite motif; UIM, ubiquitin-interaction motifs; USP11, ubiquitin-proteasome system protein 11; vRNPs, viral ribonucleoprotein complex; VSV, vesicular stomatitis virus; ZBP1, Z-DNA-binding protein 1. 


\section{INTRODUCTION}

Influenza A viruses (IAV) are enveloped viruses containing eight single-stranded, negative-sense RNA gene segments. Each viral gene encodes one or more proteins. The viral surface is decorated with the envelope proteins, i.e., HA, NA, and matrix 2 (M2). The matrix 1 (M1) provides support underneath the envelope. Individual viral ribonucleoproteins (vRNPs) complexes containing viral RNA (vRNA) from the eight gene segments reside inside each virion (Figure 1A). The vRNPs are composed of genomic RNA bound at both ends by the trimeric viral polymerase containing $\mathrm{PB} 1, \mathrm{~PB} 2$, and $\mathrm{PA}$ and coated by oligomers of NP (Figure 1B). Drug-resistant influenza viruses commonly arise as a result of frequent genetic changes, which can lead to antiviral drug resistance. Therefore, development of new effective antiviral therapies against influenza viruses is urgently needed. Owing to its relatively small genomic-coding capacity and the nature as an obligate intracellular parasite, IAV relies heavily on host cellular functions and must coopt host cellular machineries to support all stages of its life cycle, from entry to viral budding out of the infected cells. Additionally, influenza viruses either counteract or exploit different complex cellular mechanisms to establish an efficient infection and achieve survival.

Post-translational modifications (PTMs) substantially affect protein function through a wide variety of regulatory mechanisms, such as modulating protein shape, regulating cellular localization, and affecting protein interactions with other macromolecules. The functions of influenza virus proteins are finely regulated by diverse types of PTMs, most notably phosphorylation, ubiquitination, SUMOylation, and acetylation (Fu et al., 2015; Rudnicka and Yamauchi, 2016; Giese et al., 2017; Zheng et al., 2017; Dawson and Mehle, 2018; Tan and Gao, 2018). Phosphorylation is a common mechanism by which pathway cascades are modified, and it is used to control many key features, such as multiprotein assembly formation, protein stability, and enzymatic activity (Deribe et al., 2010). Ubiquitination is a reversible PTM that depends on the concerted action of ubiquitinating and deubiquitinating enzymes (Calistri et al., 2014). The modification process of SUMOylation is similar to that of ubiquitination; modifications resulting from both these processes regulate protein interactions by modifying the substrate proteins. In addition, SUMO can competitively bind the substrate proteins of ubiquitin. Both ubiquitination and SUMOylation are widely involved in various cellular processes, including DNA damage repair, transcriptional regulation, protein function regulation, and protein-protein interaction, as well as in various signaling pathways, such as those linked to gene regulation, epigenetics, cell differentiation, protein degradation, and tumorigenesis; they also play roles in viral replication and immune responses (Johnson, 2004; Pickart, 2004; Welchman et al., 2005). Acetylation is another major class of PTMs that is carried out by lysine acetyltransferases and reversed by lysine deacetylases to control various cellular and viral protein functions (Marazzi et al., 2012; Drazic et al., 2016). Other PTMs, such as glycosylation (de Vries et al., 2010; Herfst et al., 2012), NEDDylation (Hughes et al., 2015; Zhang et al., 2017), and ISGylation (Tang et al., 2010; Zhao et al., 2010) also play important roles in regulating protein function and contribute to processes involved in viral infection.

During IAV infection, host cells can accelerate the immune response by changing the functions of existing proteins via PTMs. However, viruses also use PTMs to break through these defenses, replicate efficiently, and disseminate quickly to new host cells. Many PTMs have been identified as central players in mediating the host innate immune response to influenza virus infection (Rajsbaum et al., 2012; Xing et al., 2016; Koliopoulos et al., 2018; Winkler et al., 2019). Such events are often beneficial for virus replication and/or constitute immune evasion strategies associated with efficient viral gene expression and virus dissemination in the host. Understanding how influenza virus uses PTMs system for efficient infection may identify novel targets for pharmacological intervention. Here, we discuss how IAV uses PTMs to accomplish every step of the virus replication cycle, from viral entry into the host cell, replication and transcription of the genome, nucleocytoplasmic shuttling of ribonucleoprotein components, transport of newly synthesized proteins, assembly of new particles, to budding and release from the infected cell. We also discuss how the IAV usurps the PTMs system to evade host innate immune responses, thus accelerating viral pathogenesis.

\section{PTMS INVOLVED IN THE VIRAL ENTRY PROCESS OF IAV}

To successfully enter the host cell, influenza virus goes through a multistep entry process (Edinger et al., 2014). This process has six fundamental steps: (1) The virus attaches to the cell through the binding of the envelope protein HA to the sialic acid receptor on the cell surface; (2) Upon attachment, the virus is internalized into the cell, mainly through Clathrin-mediated endocytosis (Matlin et al., 1981; Yoshimura et al., 1982); (3) The virus is transported from early endosomes to late endosomes through endosomal trafficking (Rust et al., 2004; Cocucci et al., 2012); (4) Viral and endosomal membranes fuse in a low-pH environment through a process that is HA-dependent and is essential for releasing vRNPs into the cytoplasm (Maeda and Ohnishi, 1980; Daniels et al., 1985; White and Wilson, 1987); (5) The vRNPs are released from the structured layer of M1 and then enter into the cytoplasm through a process called viral uncoating; (6) Last, vRNPs are transported into the nucleus, marking the end of the viral entry process and the beginning of the nuclear phase of the replication cycle. Most of these influenza virus entry steps depend critically on the host cells, and numerous PTMs have been identified in recent years as being instrumental during this process (Table 1).

\section{PTMs Involved in IAV Binding and Internalization}

The initial viral entry step is virion attachment to the host cell through the binding of HA with the sialic acid receptor, which is regulated by species-specific sialic acid linkages. Usually, the HA protein of avian IAV preferentially binds to sialic acids linked to galactose by an $\alpha-2,3$-linkage receptor (Sia $\alpha 2,3 \mathrm{Gal}$ ), whereas 


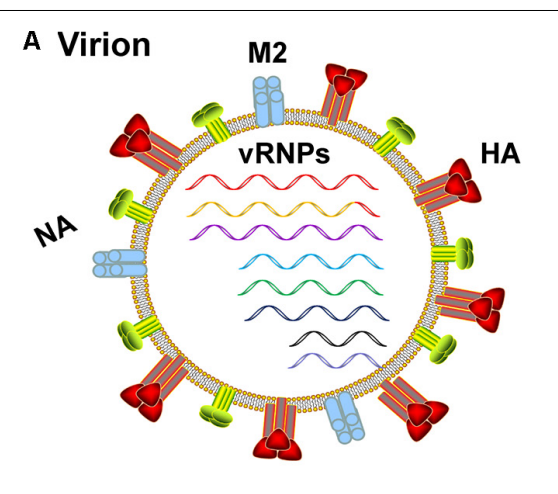

\section{B VRNPs}
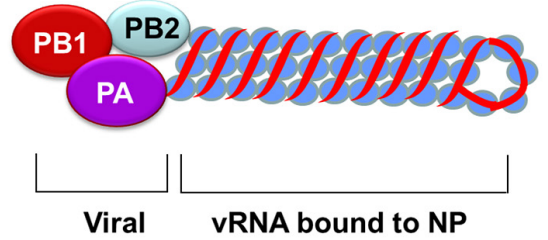

polymerase

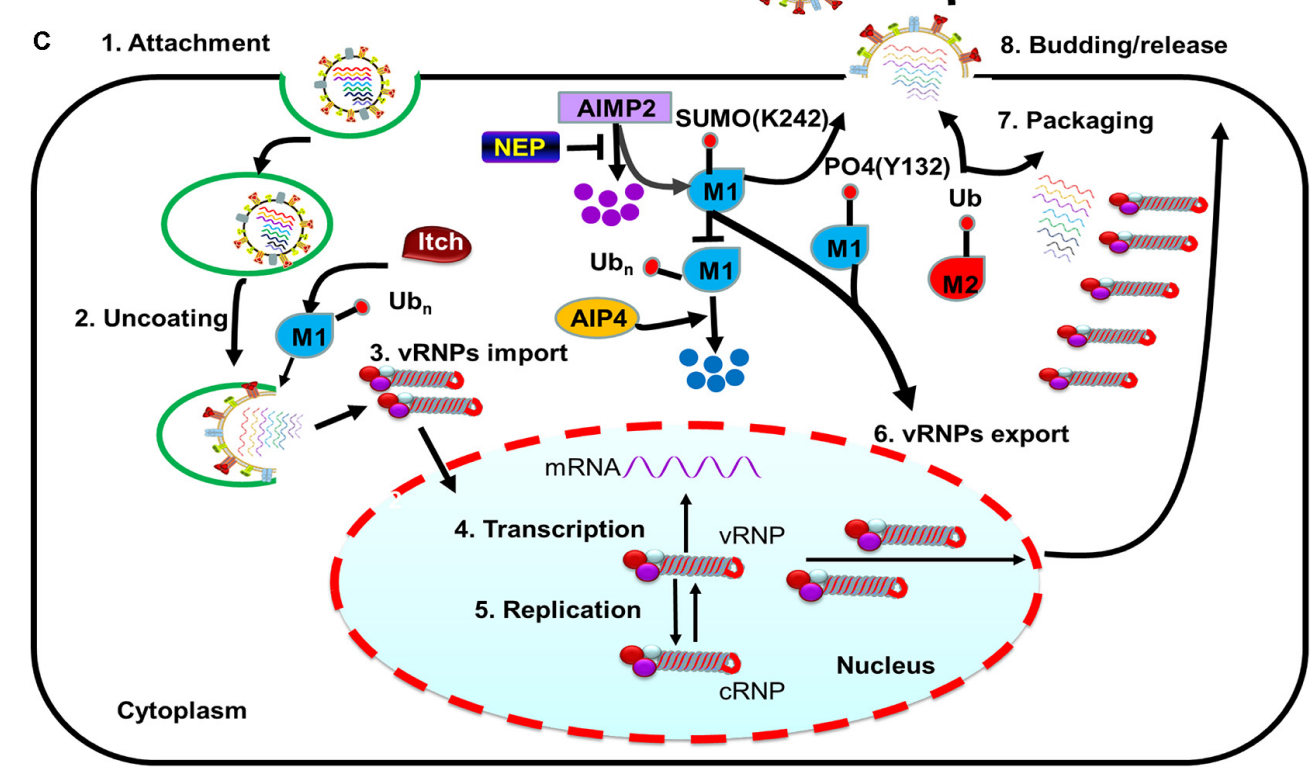

FIGURE 1 | M protein-associated PTMs that regulate the influenza virus life cycle. (A) Diagram of influenza A viruses (IAV). IAV are enveloped viruses containing eight single-stranded negative-sense RNA gene segments that each encodes one or more proteins. The viral membrane proteins HA, NA, and M2 are shown, along with eight viral ribonucleoproteins (vRNPs). (B) The structure of influenza virus vRNPs. The vRNPs of influenza virus are composed of genomic RNA (red line) bound at both ends by the trimeric viral polymerase (containing PB1, PB2, and PA) and coated by oligomers of NP (blue ball). (C) PTMs associated with M1 or M2 that regulate the influenza virus life cycle. Ubiquitination of $\mathrm{M} 1$ by the E3 ubiquitin ligase Itch promotes virus uncoating, thus facilitating vRNPs release from endosomes. M1 SUMOylation at K242 and phosphorylation at Y132 promote vRNPs export. The host protein AIMP2 changes the modification state of M1 from ubiquitinated to SUMOylated. NEP alleviates the ubiquitination of AIMP2; leading to enhanced M1 import and subsequent efficient vRNPs export. M2 ubiquitination facilitates the packaging of the viral genome. M1 SUMOylation at K242 and M2 ubiquitination at K78 contribute to viral assembly and maturation. Abbreviations: SUMO, SUMOylation; Ub, ubiquitin and ubiquitination; PO4, phosphorylation; $\mathrm{VRNP}(\mathrm{s})$, viral ribonucleoprotein(s); mRNA, messenger RNA; AIMP2, aminoacyl tRNA synthase complex-interacting multifunctional protein 2; AIP4, atrophin-1-interacting protein 4; HA, hemagglutinin; M1, viral matrix 1; M2, viral matrix 2; NA, neuraminidase; NEP, nuclear export protein.

the HA protein of human IAV shows a high affinity for Sia $\alpha 2$, 6 Gal receptor (Weis et al., 1988; Gamblin et al., 2004; Stevens et al., 2006). Interestingly, the preference for sialic acids with different terminal linkages and the affinity for sialic acid partially stem from the N-linked glycosylation status of HA (de Vries et al., 2010). Moreover, the extent of HA glycosylation may play a role in the cross-species transmission of influenza virus (Herfst et al., 2012). However, although it is generally accepted that the sialic acid receptor is the main receptor used by IAV for entry, additional host factors, such as Annexin V (Huang et al., 1996), C-type lectins (Londrigan et al., 2011), and 6-sulfo sialyl Lewis X receptors (Gambaryan et al., 2008), have also been proposed as alternative host receptors used by IAV for successful attachment and entry into the target cells.

Viral attachment to the host cell triggers virion internalization via Clathrin-mediated endocytosis (Roy et al., 2000; Rust et al., 2004; Chen and Zhuang, 2008), Clathrin-independent endocytosis (Nunes-Correia et al., 2004), or macropinocytosis (Sieczkarski and Whittaker, 2002; Rust et al., 2004; de Vries et al., 2011). The internalization of IAV through Clathrinmediated endocytosis involves Dynamin (Roy et al., 2000) and requires the adaptor protein Epsin 1 (Chen and Zhuang, 2008). 
TABLE 1 | Roles of post-translational modifications during the influenza A virus life cycle.

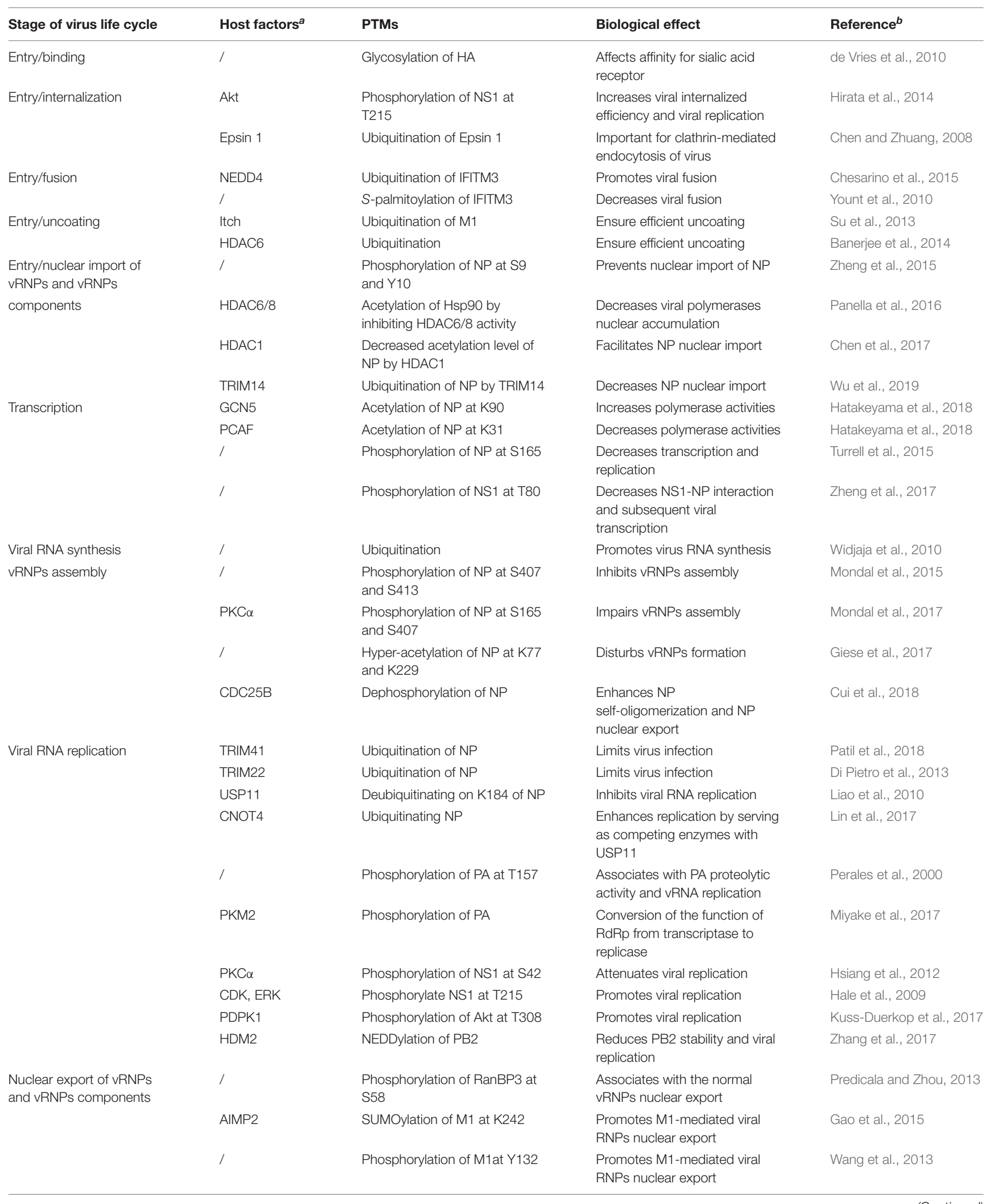


TABLE 1 | Continued

\begin{tabular}{|c|c|c|c|c|}
\hline Stage of virus life cycle & Host factors ${ }^{a}$ & PTMs & Biological effect & Reference $^{b}$ \\
\hline & / & Phosphorylation of NP at Y296 & $\begin{array}{l}\text { Obstructs nuclear export of } \\
\text { vRNPs by inhibiting NP binding } \\
\text { to CRM1 }\end{array}$ & Zheng et al., 2015 \\
\hline & / & Phosphorylation of MLC & $\begin{array}{l}\text { Promotes RNP complex } \\
\text { nuclear export }\end{array}$ & Haidari et al., 2011 \\
\hline & / & Phosphorylation of NP at T188 & $\begin{array}{l}\text { Impedes nuclear export signal } \\
\text { 2-dependent NP nuclear export }\end{array}$ & Li et al., 2018 \\
\hline $\begin{array}{l}\text { Cytoplasmic transport of } \\
\text { vRNPs }\end{array}$ & HDAC6 & Acetylation of $\alpha$-tubulin & $\begin{array}{l}\text { Downregulates the trafficking of } \\
\text { viral RNPs to the host cell } \\
\text { plasma membrane }\end{array}$ & Husain and Cheung, 2014 \\
\hline \multirow[t]{2}{*}{ Assembly/packaging } & / & Ubiquitination of M2 at K78 & $\begin{array}{l}\text { Facilitates the packaging of the } \\
\text { viral genome into virus particles }\end{array}$ & Su et al., 2018 \\
\hline & / & Palmitoylation of HA & $\begin{array}{l}\text { Correlates with assembly and } \\
\text { infectious influenza virus } \\
\text { particles formation }\end{array}$ & Chen et al., 2005 \\
\hline \multirow[t]{3}{*}{ Virus budding/release } & / & SUMOylation of M1 at K242 & $\begin{array}{l}\text { Promotes virus release by } \\
\text { facilitating M1-vRNPs complex } \\
\text { formation }\end{array}$ & Wu et al., 2011 \\
\hline & / & Acetylation of microtubules & Increases virus release & Husain and Harrod, 2011 \\
\hline & / & $\begin{array}{l}\text { Hyper-acetylation of NP at } \\
\text { K229 }\end{array}$ & Impairs viral particle release & Giese et al., 2017 \\
\hline
\end{tabular}

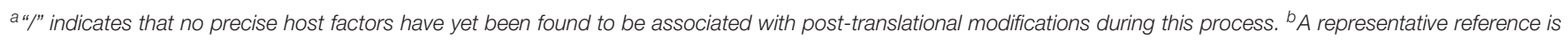
listed. Please see the text for more details.

The UIM of Epsin 1 plays a critical role in the Clathrinmediated endocytosis of influenza virus (Chen and Zhuang, 2008) (Table 1). Non-structural protein 1 (NS1) can interact with Akt, a core intracellular survival regulator and the major effector molecule of phosphoinositide 3-kinase (PI3K). Suppression of Akt kinase activity decreases the phosphorylation levels of NS1 and glycogen synthase kinase 3 (Akt substrate), leading to reduced viral internalization efficiency and viral replication (Hirata et al., 2014) (Table 1).

\section{PTMs Involved in IAV Endosome Trafficking and Fusion}

Upon influenza virus internalization by either the endocytosis or macropinocytosis uptake pathway, endosomal trafficking from early endosomes to late endosomes begins. IFITM3 can localize to endosomes, acting as a viral fusion inhibitor and exerting a broad-spectrum antiviral activity. However, the E3 ubiquitin ligase NEDD4 can alleviate IFITM3-mediated inhibition of fusion by decreasing IFITM3 expression via ubiquitination (Chesarino et al., 2015) (Table 1). In contrast, S-palmitoylation promotes the antiviral activity of IFITM3, mainly through manipulating its clustering in membranes and blocking influenza virus fusion (Dawson and Mehle, 2018).

\section{PTMs Involved in Uncoating and Nuclear Import of IAV vRNPs and vRNP Components}

After HA-mediated membrane fusion occurs in late endocytic vacuoles, the viral capsids are released from the cytosolic surface of endosomes, the vRNPs and the M1 protein then dissociate from each other and disperse in the cytosol, and the vRNPs are imported into the nucleus through nuclear pore complexes (Martin and Helenius, 1991; O’Neill et al., 1995). During this process, IAV takes advantage of the host cellular ubiquitindependent aggresome formation and disassembly machinery (Banerjee et al., 2014). The unanchored ubiquitin chains, which are packaged into virions, can facilitate viral uncoating by recruiting histone deacetylase (HDAC) 6 to viral fusion sites via the zinc-finger ubiquitin-binding domain (ZnFUBP) of HDAC6 (Banerjee et al., 2014). Itch, an E3 ubiquitin ligase, also enables efficient uncoating via ubiquitinating M1, thereby facilitating vRNPs release from the endosomes (Su et al., 2013) (Table 1 and Figure 1). Released vRNPs then move toward the nucleus to complete the last step of viral entry (vRNPs nuclear import) and begin viral transcription. Currently, very few PTMs have been shown to directly regulate the localization of viral polymerases. However, the inhibition of HDAC6/8 activity by HDAC-inhibitor MC1568 resulted in the early acetylation of HSP90 and led to a decrease in viral polymerases nuclear accumulation and a subsequent attenuated viral replication (Panella et al., 2016) (Table 1). Nuclear import of the vRNP components, such as NP, is finely regulated by phosphorylation, SUMOylation, and acetylation. Phosphorylation at S9 or Y10 in the nuclear localization signal (NLS) of NP prevents its nuclear import by regulating the association of this protein with its nuclear import receptors, such as Importin- $\alpha$ (Zheng et al., 2015) (Figure 2). The NP-interacting protein TRIM14 stimulates K48-linked ubiquitination and proteasomal degradation of NP, leading to a reduced efficiency in NP transportation from the cytoplasm to the nucleus and a subsequent inhibition of viral replication (Wu et al., 2019) (Table 1 and Figure 2). Meanwhile, HDAC1 interacts with NP, resulting in a decreased level of NP acetylation, thus facilitating viral replication through promoting 

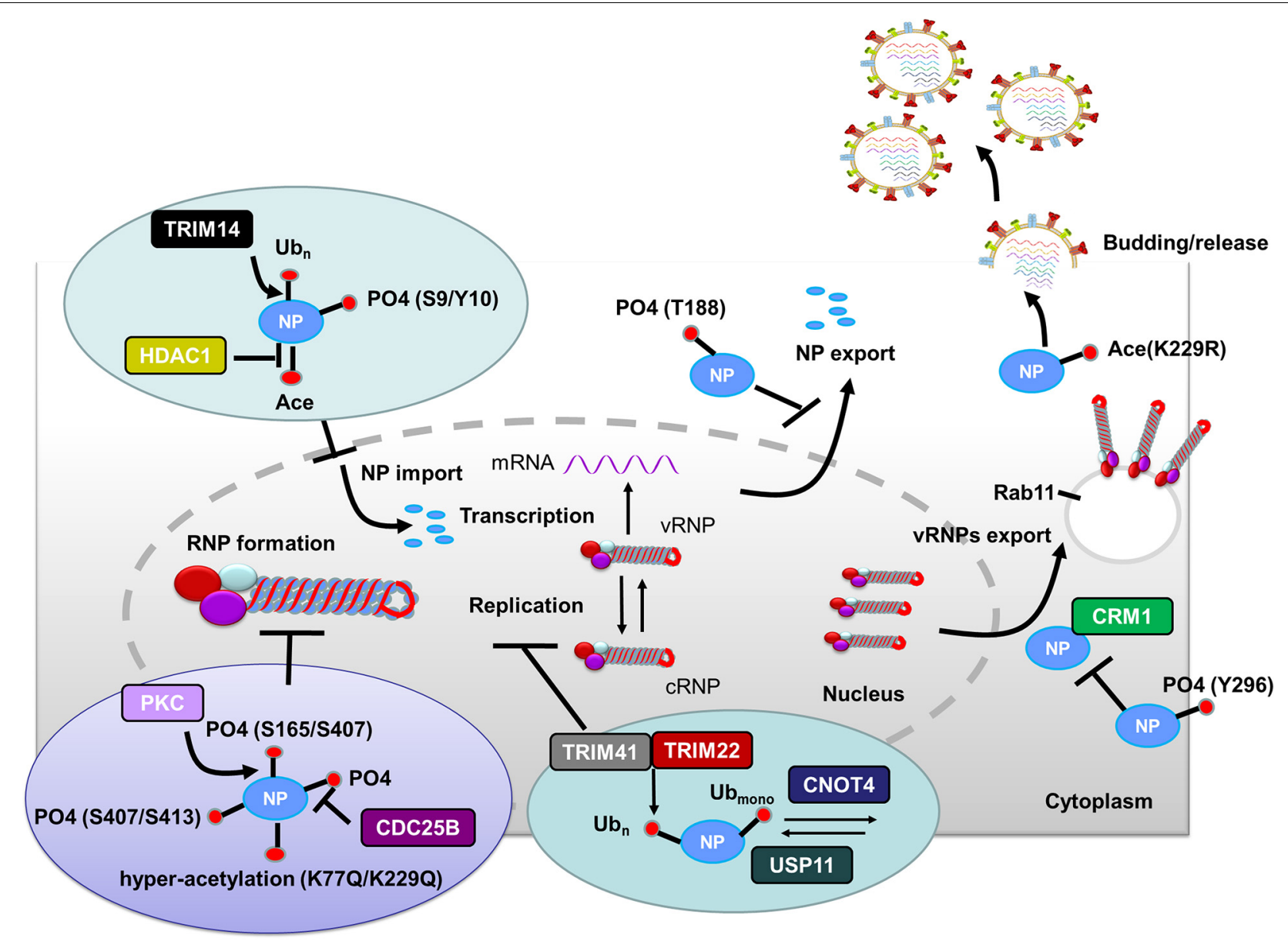

FIGURE 2 | Role of PTMs in regulating NP functions. Phosphorylation of NP at S9 and Y10 and ubiquitination of NP by TRIM14 prevent NP nuclear import. HDAC decreases NP acetylation and facilitates NP nuclear import. Phosphorylation of NP at S407 and S413 inhibits the assembly of influenza virus replication machinery. Mimicking hyperacetylation of NP at K77Q and K229Q disturbs vRNPs formation. Human PKC $\alpha$ blocks NP oligomerization through phosphorylating NP at S165 and S407. In contrast, CDC25B facilitates the dephosphorylation of NP, leading to enhanced NP self-oligomerization. TRIM41 and TRIM22 impede viral replication through ubiquitinating NP. The cellular deubiquitinating enzyme USP11 inhibits VRNA replication through deubiquitinating NP K184; CNOT4 competes with USP11 in the regulation of NP ubiquitination and enhances VRNA replication. Phosphorylation of NP Y296 impedes VRNPs nuclear export via inhibiting the interaction of NP with the export factor CRM1. Phosphorylation of NP T188 impedes NP nuclear export. Exported VRNPs associate with Rab11-positive vesicles and traffic from the cytoplasm to the apical cell surface for virion budding. NP acetylation plays a role in virion release. Abbreviations: Ub, ubiquitin and ubiquitination; PO4,

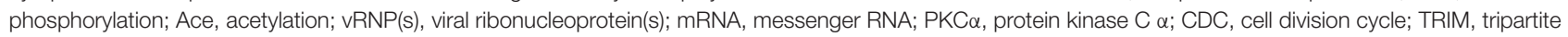
motif; HDAC, histone deacetylase; USP11, ubiquitin-proteasome system protein 11; CNOT4, Ccr4-Not transcription complex subunit 4; CRM1, chromosome region maintenance 1 protein homolog.

the nuclear import of NP and suppressing the TBK1-IRF3 signal pathway (Chen et al., 2017) (Table 1 and Figure 2).

\section{PTMS INVOLVED IN THE VIRAL TRANSCRIPTION AND REPLICATION OF IAV}

The replication of IAV RNA requires an intricate regulatory network composed of both viral and cellular proteins. IAV uses distinctly different strategies for transcription and replication (Barrett et al., 1979). For transcription, 5'-capped oligonucleotides derive from cellular mRNAs by cap-snatching are used as primers. In contrast, no primers are needed for replication. The vRNA template is directly copied to form a full-length positive-stranded RNA (cRNA), which serves as the template for vRNA synthesis (Hay et al., 1982). Moreover, another major difference between transcription and replication is that influenza virus RNA replication requires newly synthesized viral proteins. PTMs of viral proteins and host factors play important roles in regulating influenza virus replication. Here, we summarize the PTMs involved in the process of producing viral transcripts and new copies of the viral genome.

\section{PTMs Involved in IAV Transcription}

Influenza virus uses a unique strategy for priming transcription, referred to as "cap-snatching," where short 5'-fragments containing 7-methylG caps are cleaved from host messenger 
RNAs (mRNAs) and used to prime the synthesis of viral mRNAs (Plotch et al., 1981). To complete the cap-snatching process, the transcribing polymerases must gain access to the nascent host mRNA through binding to cellular RNA polymerase II (RNAPII) and selectively recognizing PTM patterns in its C-terminal domain (CTD) (i.e., phosphorylated CTD) (Engelhardt et al., 2005).

The acetylation and phosphorylation of NP are actively involved in regulating influenza viral transcription. The host acetyltransferase GCN5 can acetylate NP K90, leading to increased IAV polymerase activities, whereas acetylation of NP K31 mediated by PCAF decreases polymerase activities (Hatakeyama et al., 2018) (Table 1). The phosphorylation of NP can stimulate viral transcription, and NP must undergo specific conformational changes to ensure efficient transcriptional activity (Kamata and Watanabe, 1977). As a PB1-interacting protein, the E3 ubiquitin ligase TRIM32 directly limits viral infection in mice by targeting PB1 for ubiquitination, which downregulates viral polymerase activity (Fu et al., 2015). This intrinsic antiviral defense strategy provides a novel target for drugs to prevent and treat influenza. PKC $\alpha$ is a multifunctional effector in influenza virus-induced signaling cascades. PKC $\alpha$ mediates the phosphorylation of PB1 and NS1, consequently contributing to the activity of vRNA polymerase complexes (Mahmoudian et al., 2009). Moreover, the phosphorylation of NS1 T80 reduces IAV replication both in vitro and in vivo through two mechanisms: (1) decreasing NS1-NP interactions, leading to impaired vRNPs-mediated viral transcription, and (2) reducing the binding affinity between NS1 and RIG-I, resulting in the virus being unable to inhibit IFN production (Zheng et al., 2017).

\section{PTMs Involved in IAV vRNA Synthesis}

Influenza virus replication depends on the ubiquitin-proteasome system. Several studies have preliminarily reported an important role of ubiquitination in virus entry (Khor et al., 2003; Chen and Zhuang, 2008) and vRNA synthesis (Konig et al., 2010). However, there remains a lack of direct evidence for this phenomenon. Widjaja et al. used both proteasome inhibitors (MG132 and bortezomib) and the E36ts20 hamster cell line harboring a temperature-sensitive mutation in the E1-activating enzyme to investigate the detailed role of the ubiquitin-proteasome system in IAV replication cycle (Widjaja et al., 2010). Their results indicate that ubiquitination efficiently promotes vRNA synthesis and subsequent protein expression (Widjaja et al., 2010).

\section{PTMs Involved in IAV Viral Genome Replication and vRNPs Assembly}

The vRNPs complex contains the viral polymerase, genomic RNA, and NP. The NP protein exists in an oligomeric form consisting of multiple copies, which bind to and coat the viral RNA genome. Before vRNPs complex assembly begins, the virus must complete primary transcription and transition to genome replication. During the vRNPs assembly process, an NP monomer is initially recruited through direct interaction with viral polymerase. After binding to the nascent 5 ' end of the viral genome, monomeric NP oligomerizes along the length of the genomic RNA via NP-NP homo-oligomerization (Vreede et al., 2004; Ye et al., 2006; Tao and Ye, 2010; Arranz et al., 2012; Moeller et al., 2012). Phosphorylation of NP inhibits the assembly of influenza virus replication machinery. Mechanistically, two phosphorylation sites (S407 and S413) on the opposite sides of the NP-NP interface block homotypic interactions and destroy the balance between NP monomers and oligomers, thus maintaining the monomeric form of NP (Mondal et al., 2015) (Figure 2). Human PKC $\alpha$ impairs vRNPs assembly through phosphorylating $\mathrm{NP}$ at the tail loop-groove interface (major targets: S165 and S407), thus blocking NP oligomerization and vRNPs formation in cells (Mondal et al., 2017) (Table 1 and Figure 2). In addition, mimicking hyperacetylation of NP at K77Q and K229Q disturbs vRNPs formation and severely diminishes viral polymerase activity (Giese et al., 2017) (Table $\mathbf{1}$ and Figure 2). S165 is present within the NP homo-oligomerization domain. It was proposed that reversible phosphorylation of NP S165 decreases NP oligomerization prior to vRNPs assembly and inhibits mRNA and vRNA accumulation, resulting in subsequent attenuation of viral growth in Madin-Darby bovine kidney (MDBK) cells (Turrell et al., 2015). CDC25B, a member of the CDC25 phosphatase family, facilitates the dephosphorylation of NP, leading to enhanced self-oligomerization and nuclear export of NP along with increased viral polymerase activity and vRNA production (Cui et al., 2018) (Table 1 and Figure 2).

The phosphorylation of other proteins also plays a role in influenza virus replication. For example, the phosphorylation of PA is highly associated with its proteolytic activity, which decreases the expression levels of both PA and its co-expressed proteins (Hara et al., 2001, 2003). Casein kinase II can target 11 potential phosphorylation sites on PA. Among these sites, a T157A mutation was found to cause defective phosphorylation and almost completely abrogate proteolysis activity, leading to sharply reduced vRNA replication (Perales et al., 2000) (Table 1). Pyruvate kinase M2 (PKM2), an important enzyme for ATP generation (Altenberg and Greulich, 2004), can interact with PA. PKM2 positively regulates viral replication possibly through phosphorylation of $\mathrm{PA}$, which leads to RdRp switching its function from a transcriptase to a replicase (Miyake et al., 2017). Furthermore, phosphorylation of NS1 S42 catalyzed by PKC $\alpha$ attenuates human IAV replication (Hsiang et al., 2012). Cyclin-dependent kinases (CDKs) and extracellular signalregulated kinases (ERKs) can phosphorylate NS1 T215 and contribute to efficient virus replication (Hale et al., 2009). Moreover, NS1 can induce $\mathrm{G}_{0} / \mathrm{G}_{1}$ cell cycle arrest by interfering with the RhoA-pRb signaling cascade through downregulating the phosphorylation level of cell cycle regulator $\mathrm{pRb}$, thus providing favorable conditions for viral protein accumulation and replication (Jiang et al., 2013).

The host kinase mechanistic target of rapamycin (mTOR), a cellular regulator of protein synthesis, growth, and motility, is coopted by influenza virus to promote infection (Konig et al., 2010; Mata et al., 2011). To maximize viral replication during the late stages of infection, influenza virus differentially activates the signaling pathways of mTOR complex 1 and 2 (mTORC1 and mTORC2, respectively) that are two functionally 
distinct multiprotein complexes (Kuss-Duerkop et al., 2017). Influenza virus replication and $\mathrm{HA}$ can ensure efficient viral replication by enhancing $\mathrm{mTORC1}$ activation through PDPK1mediated phosphorylation of Akt at T308. Influenza virus M2 then further promotes viral replication by downregulating the expression of REDD1 (an mTORC1 inhibitor) to support mTORC1 activation. Finally, NS1 promotes mTORC2-mediated phosphorylation of Akt at S473 and subsequently enhances cell apoptosis (Kuss-Duerkop et al., 2017).

Ubiquitination contributes to proteasomal degradation, and all the proteins in the influenza virus replication machinery are ubiquitinated (Kirui et al., 2016). Most of the NP ubiquitinations are associated with viral replication. TRIM protein superfamily members are emerging as crucial antiviral effectors, which often rely on their E3 ubiquitin ligase activities to restrict influenza viral replication. TRIM41 acts as an intrinsic host restriction factor for IAV, limiting viral infection by directly binding to NP, leading to its ubiquitination and subsequent proteasomal degradation (Patil et al., 2018) (Figure 2). TRIM22, another NP-interacting protein, is highly upregulated in human alveolar epithelial A549 cells and also acts to restrict IAV replication by degrading viral NP via its ubiquitination (Di Pietro et al., 2013) (Figure 2).

Deubiquitination of NP can also affect influenza viral replication. The cellular deubiquitinating enzyme USP11 inhibits vRNA replication through deubiquitinating NP K184 to lower the NP binding affinity for cRNA (Liao et al., 2010). CNOT4, a cellular ubiquitin ligase, competes with USP11 in the regulation of NP ubiquitination, and thereby enhancing vRNPs activity and vRNA replication (Lin et al., 2017) (Table 1 and Figure 2). Thus, NP ubiquitination has both proviral and antiviral roles, and the ubiquitination and deubiquitination of NP must be properly balanced for efficient influenza infection.

NEDDylation is another important modification that regulates protein activity and protein-protein interactions. It has been shown to play important roles during the life cycles of various viruses, such as human immunodeficiency virus type-1 (HIV-1) and Kaposi's sarcoma-associated herpesvirus (Esposito et al., 2009; Soucy et al., 2010; Stanley et al., 2012; Hughes et al., 2015). Influenza virus PB2 can be NEDDylated at residue K669 by NEDDylation E3 ligase HDM2, leading to reduced PB2 stability and a subsequent weakened viral replication and virulence in mice (Zhang et al., 2017).

\section{PTMS INVOLVED IN IAV NUCLEAR EXPORT, TRAFFICKING, AND BUDDING}

In the later steps of the influenza viral replication cycle, newly synthesized vRNPs are exported out of the nucleus and trafficked to the budding site at the plasma membrane, where they are assembled into virions and ultimately bud from the infected cell (Lakdawala et al., 2016). At the same time, the influenza virus structural proteins NA, HA, M1, and M2 are processed and accumulate at the virion assembly sites on the plasma membrane. Knowledge of how PTMs contribute to these processes has been growing quickly in recent years. In the following section, we will review the current progress regarding the involvement of PTMs in the later steps of the influenza virus life cycle (Table 1).

\section{PTMs Involved in the Nuclear Export of IAV vRNPs and vRNP Components}

Newly synthesized vRNPs are exported out from the nucleus through the nuclear pore complex, which relies on host cellular export machinery. CRM1 is the major exporting factor responsible for the transport of influenza virus vRNPs from the nucleus to the cytoplasm. During this process, M1 plays an important role in facilitating the interaction between vRNPs and the host cellular export system. Specifically, vRNPs can interact with M1, M1 can interact with nuclear export protein (NEP), and NEP can interact with CRM1 (Paterson and Fodor, 2012). Therefore, M1 likely acts as an adapter protein between vRNPs, NEP, and the host cellular export system. M1 with phosphorylation modification plays a concerted role in promoting the nuclear export of vRNPs during influenza virus infection (Bui et al., 2000). Additionally, AIMP2, an NS1-interacting protein, enhances M1 stability and changes the modification state of M1 K242 from ubiquitinated to SUMOylated, thus promoting M1-mediated vRNPs nuclear export (Gao et al., 2015) (Table 1 and Figure 1). Phosphorylation of M1 Y132 is essential for influenza virus replication, which controls the nuclear import of M1 and the subsequent nuclear export of progeny vRNPs by regulating the interaction between M1 and the nuclear import factor importin-1 (Wang et al., 2013) (Figure 1). Moreover, SUMOylation of M1 also enhances its interactions with vRNPs and promotes the subsequent nuclear export of vRNPs (Wu et al., 2011).

Phosphorylation of NP Y296 obstructs the nuclear export of vRNPs complexes and subsequently impairs virus assembly and polymerase activity through inhibiting the binding affinity of NP to export factor CRM1 (Zheng et al., 2015) (Figure 2). RanBP3 is a Ran-interacting protein that acts as a nuclear export cofactor of CRM1-mediated cargo during influenza virus infection. Phosphorylation of RanBP3 at S58 is associated with normal vRNPs nuclear export, and downregulation of RanBP3 expression impairs the nuclear export of influenza virus vRNPs (Predicala and Zhou, 2013).

The actin and microtubule cytoskeleton also play a critical role in viral replication (Radtke et al., 2006). Actin cytoskeleton contraction and relaxation are predominantly regulated by phosphorylation and dephosphorylation of the regulatory subunit of MLC (Lincoln, 2007). Influenza virus infection activates a series of signaling pathways that induce MLC phosphorylation and actin cytoskeleton remodeling. The inhibition of MLC phosphorylation leads to nuclear retention of influenza vRNPs complexes and a reduction of influenza virus replication, whereas the induction of MLC phosphorylation reverses the inhibitory effects of unphosphorylated MLC on the nuclear translocation of influenza virus vRNPs complexes (Haidari et al., 2011). Nuclear export of vRNPs requires sufficient amounts of $\mathrm{HA}$ and NA to be embedded in the plasma membrane, and this process is coordinated in part by $\mathrm{PKC} \alpha /$ mitogen-activated protein (MAP) kinase signaling cascade 
mediated by HA membrane accumulation (Pleschka et al., 2001; Marjuki et al., 2006). Blocking this pathway during influenza virus infection leads to vRNPs nuclear retention, impaired nuclear-export protein (NEP/NS2) function, and concomitant inhibited virus propagation (Pleschka et al., 2001; Marjuki et al., 2006).

The NP protein has an intrinsic ability to be exported from the nucleus to the cytoplasm, and the nuclear export ability of NP is partially regulated by phosphorylation. A lack of NP phosphorylation may block NP nuclear export or allow a rapid reimportation of NP into the nucleus (Neumann et al., 1997). Phosphorylation of NP T188 impedes NES2-dependent NP nuclear export, decreases viral polymerase activity as well as viral replication (Li et al., 2018) (Figure 2).

\section{PTMs Involved in IAV Protein Trafficking to the Plasma Membrane}

Influenza virus assembles at the host cell plasma membrane. Before budding, the newly synthesized vRNPs and viral membrane proteins (HA, NA, and M2) are translocated to the budding site at the plasma membrane via different trafficking systems. To reach the budding site, the vRNPs mainly use the microtubule network and vesicular transport system (Hutchinson and Fodor, 2013), whereas HA, NA, and M2 use the ER-Golgi secretory network. Microtubules are the largest cytoskeleton component, and they play a crucial role in a variety of cellular functions, including intracellular transport, organelle positioning, cell shape and motility, and centrosome and cilium formation (Janke and Bulinski, 2011). To accomplish such diverse functions, microtubules associate with motor and non-motor proteins and undergo posttranslational modifications such as acetylation and detyrosination (Janke and Bulinski, 2011). During influenza virus infection, downregulation of tubulin deacetylase and HDAC6 activity enhances $\alpha$-tubulin acetylation, subsequently facilitating virion release (Husain and Harrod, 2011). Mechanistically, HDAC6 downregulates the trafficking of vRNPs to the host cell plasma membrane via reducing the amount of acetylated microtubules (Husain and Cheung, 2014) (Table 1).

\section{PTMs Involved in IAV Assembly, Budding, and Release}

M2 ubiquitination plays an important role in influenza virus replication through facilitating the packaging of viral genome into virus particles and coordinating the timing of virus-induced host cell apoptosis and autophagy (Su et al., 2018) (Figure 1). Moreover, ubiquitination-deficient mutant M2 K78R, had lower infectivity because it produced mainly defective virion particles that either lacked vRNPs or contained smaller amounts of internal viral components (Su et al., 2018) (Figure 1).

Influenza A viruses assembly requires the coordinated localization of different viral components at virus budding sites. M1 can interact with vRNPs, thus playing a key role in influenza virus assembly (Schmitt and Lamb, 2005). M1 SUMOylation at K242 is required for efficient interaction between M1 and vRNPs to form the M1-vRNP complex, thus contributing to viral maturation and assembly (Wu et al., 2011). Viruses carrying a
SUMO-deficient M1 produce fewer infectious particles and form virions with more elongated or filamentous structures, indicating that a lack of M1 SUMOylation impairs viral morphogenesis (Wu et al., 2011). M1 also interacts with the viral envelope proteins HA, NA, and M2 via their cytoplasmic tails. Palmitoylation of HA at the conserved carboxy-terminal of the cytoplasmic tail is required for efficient M1 recruitment and correlates with the ability to form infectious influenza virus particles (Zurcher et al., 1994; Chen et al., 2005). Acetylation is another PTM known to regulate protein function. Enhanced acetylation of microtubules increases IAV release from infected cells (Husain and Harrod, 2011). Furthermore, the growth of NP acetylationdeficient K229R mutant was severely attenuated in various cell types due to impaired virion release (Giese et al., 2017) (Figure 2).

\section{ROLE OF PTMS IN THE ANTIVIRAL INNATE IMMUNE RESPONSE TO IAV}

Innate immunity acts as the first-line defense of host cells in restricting IAV replication (Iwasaki and Pillai, 2014). During virus infection, the pattern recognition receptors (PRR) recognize pathogen-associated molecular patterns (PAMPs) in the invading viruses, leading to the activation of innate immune signaling cascades and the subsequent production of proinflammatory cytokines (Iwasaki and Pillai, 2014). PTMs are involve in regulating innate immune signaling, and many viruses have evolved various mechanisms to usurp PTMs to counteract this host defense response (Calistri et al., 2014). For influenza virus, the activities of the immune-regulatory proteins, including NS1 (Nogales et al., 2018) and the accessory proteins PA-X (Levene and Gaglia, 2018; Nogales et al., 2018) and PB1-F2 (Cheung et al., 2020), are finely modulated by various PTMs to antagonize host innate immune responses.

\section{Role of PTMs in IAV NS1 Antagonism of Host Antiviral Innate Immune Responses}

Production of type I IFNs, a family of anti-viral cytokines, is an integral component of the host innate immunity defense system, which play a critical role in inhibiting the accomplishment of the virus life cycle and impeding virus dissemination in vivo (Garcia-Sastre et al., 1998). Influenza virus NS1 counters the IFN antiviral responses mainly through sequestering RNAs that are sensed to trigger signaling cascade activation, or interfering with the vRNA sensors themselves, particularly RIG-I and its activator TRIM25. Generally, ubiquitination by TRIM25 of the vRNA sensor RIG-I CARD with K63-linked poly-ubiquitin chains leads to IFN production. However, this process can be blocked through the interaction of NS1 E96 and E97 with TRIM25 coiled-coil-PRYSPRY domain (Gack et al., 2009). Mechanistically, NS1 binding affects the correct positioning of the TRIM25 PRYSPRY domain that is required for substrate ubiquitination, thus suppressing RIG-I ubiquitination and subsequent downstream signaling (Koliopoulos et al., 2018). Moreover, NS1 targets TRIM25 in a species-specific manner to inhibit RIG-I ubiquitination and antiviral IFN production (Rajsbaum et al., 2012) (Figure 3). Specifically, NS1 derived 


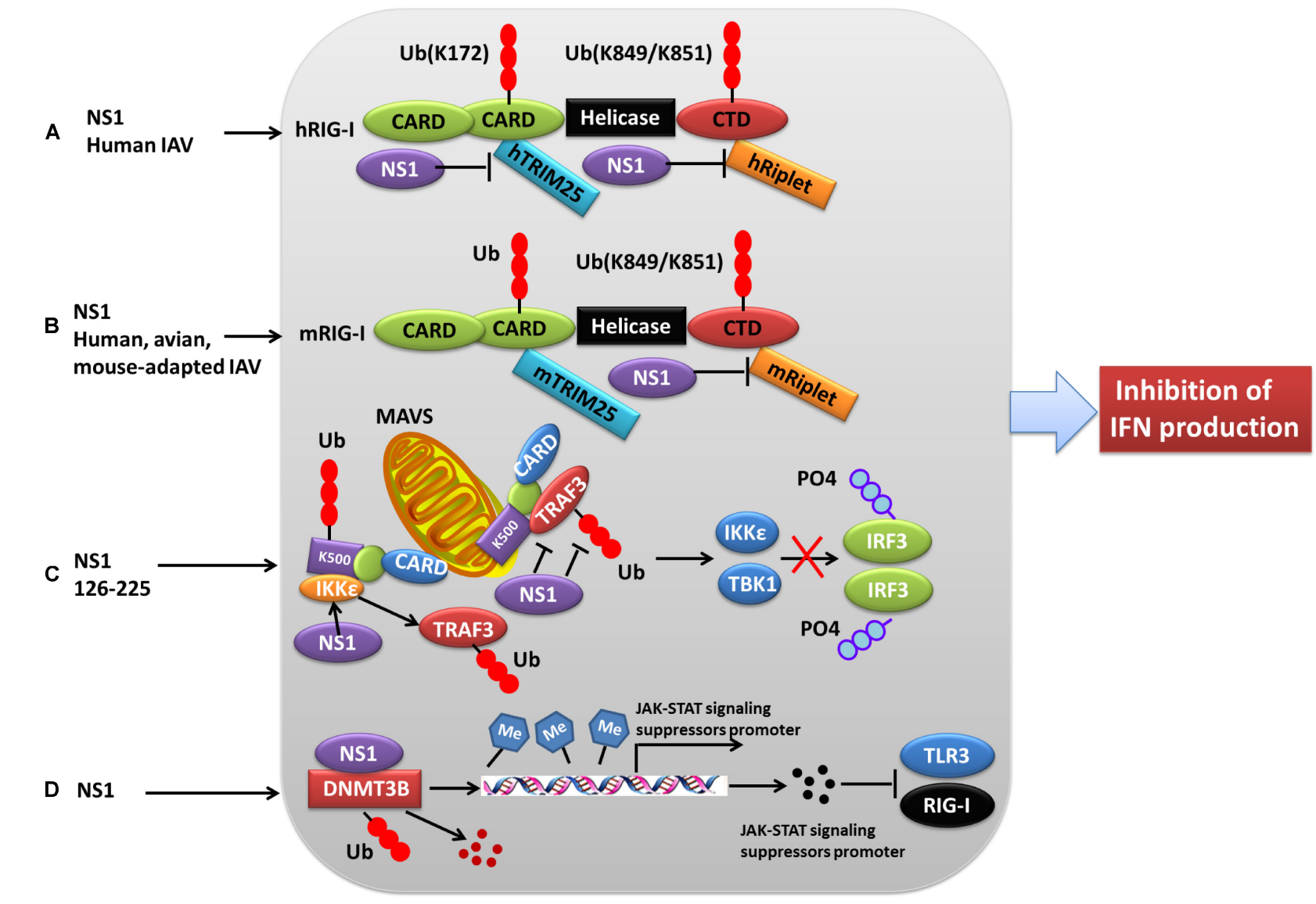

FIGURE 3 | Role of PTMs in NS1 antagonism of host interferon responses. (A) NS1 from human IAV strains can interact with human TRIM25 and Riplet, blocking them from mediating the ubiquitination of the vRNA sensor RIG-I CARD, and this eventually leads to the inhibition of IFN production. (B) NS1 proteins from avian, human, and mouse-adapted influenza viruses can inhibit RIG-I signaling in mouse cells only through binding to and blocking mouse Riplet. (C) The NS1 C-terminal effector domain (NS1 126-225) associates with TRAF3, promoting the disassociation of TRAF3 from MAVS and leading to a decreased level of K63-linked ubiquitination of TRAF3. Moreover, NS1 126-225 facilitates the recruitment of IKKE to MAVS, resulting in TRAF3 release from the mitochondria and impaired IRF3 phosphorylation. (D) NS1 interacts with cellular DNMT3B, and the resulting NS1-DNMTcomplex is translocated from the nucleus to the cytosol where it undergoes K48-linked polyubiquitination of DNMT3B. This leads to demethylation of the methylated promoters of JAK-STAT signaling suppressors and the subsequent expression of associated proteins, ultimately resulting in the inhibition of TLR3 and RIG-I. Ub, ubiquitin and ubiquitination; PO4, phosphorylation; TRIM, tripartite motif; RIG-I, retinoic acid-induced gene 1 protein; CARD, caspase recruitment domain; DNMT3B, DNA methyltransferase; TRAF3, TNF receptor-associated factor 3.

from human influenza viruses binds to and suppresses both human TRIM25 and Riplet-mediated RIG-I CARD and CTD ubiquitination (Figure 3A). In contrast, NS1 from avian, human, and mouse-adapted influenza viruses inhibits RIG-I signaling in mouse cells only through binding to and blocking mouse Riplet (Figure 3B) (Rajsbaum et al., 2012). These findings for NS1 partly explain the host adaptation ability of influenza virus.

NS1 can also antagonize the IFN- $\beta$ response via NS1 126225 binding to the TRAF domain of TRAF3, which suppresses TRAF3 K63-linked ubiquitination (Qian et al., 2017) (Figure 3C). Another mechanism by which NS1 antagonizes the host IFN response during influenza virus infection is modulation of JAK-STAT signaling (Liu S. et al., 2019). Mechanistically, the newly expressed NS1 migrates to the nucleus where it interacts with DNMT3B, and then the NS1-DNMTcomplexes are transported from the nucleus to the cytosol where they undergo K48-linked polyubiquitination of the DNMT3B. This leads to a demethylation of the methylated promoters for the JAK-STAT signaling suppressor, thus contributing to the subsequent inhibition of TLR3 and RIG-I activity (Liu S. et al., 2019) (Figure 3D).

In contrast, some PTMs negatively regulate the ability of NS1 to counteract the host IFN response, thus contributing to virus attenuation. During the later stages of influenza virus infection, the phosphorylation of NS1 at T49 leads to a reduced interaction with double-stranded RNA (dsRNA), TRIM25, and RIG-I, resulting in a defective suppression of IFN induction (Kathum et al., 2016) (Figure 4B). Moreover, as the infectious cycle progresses, dynamic phosphorylation of NS1 at T49 may alter NS1 activity (Hsiang et al., 2012). NS1 phosphorylated at T80 also fails to inhibit IFN production due to its reduced binding affinity with RIG-I, which impedes influenza virus replication (Zheng et al., 2017) (Figure 4C). Additionally, phosphorylation of NS1 at S42 by PKC $\alpha$ can block the binding of NS1 to dsRNA 


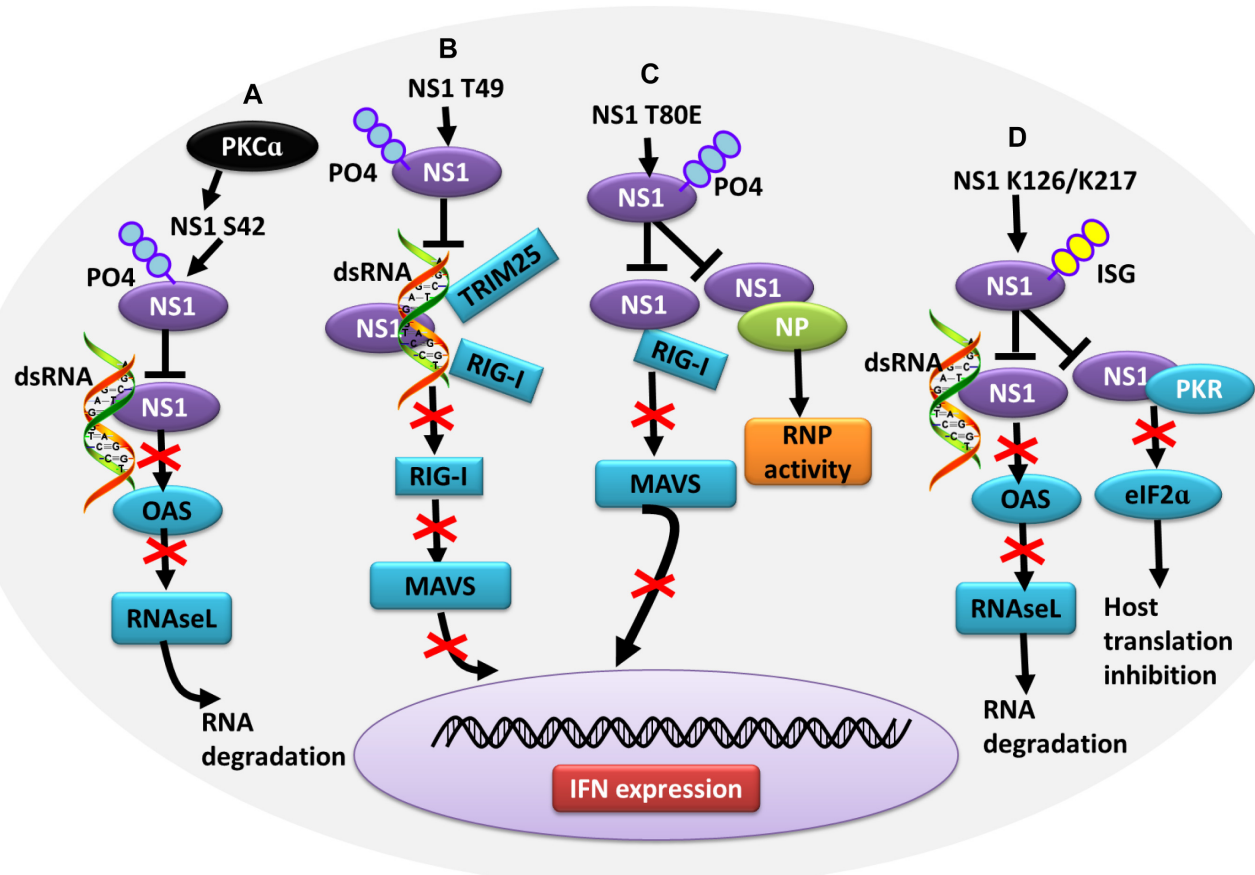

FIGURE 4 | Role of PTMs in alleviating NS1 antagonism of the host antiviral response. (A) Phosphorylation of NS1 at S42 by PKC $\alpha$ can block NS1 binding to dsRNA, alleviating NS1-induced inhibition of OAS activation and RNA degradation. (B) Phosphorylation of NS1 at T49 leads to decreased interaction with dsRNA, TRIM25, and RIG-I, resulting in defective suppression of IFN production. (C) Phosphorylation of NS1 at T80 reduces the binding affinity between NS1 and RIG-I, preventing NS1 from inhibiting IFN production. (D) Herc5-mediated ISGylation of NS1 K126 and K217 inhibits RNA sequestration, alleviating NS1-induced inhibition of OAS activation and RNA degradation. ISGylation of NS1 K126 and K217 impair the NS1 interaction with PKR, which antagonizes NS1-mediated inhibition of host translation. Ub, ubiquitin and ubiquitination; ISG, ISGylation; PKC $\alpha$, protein kinase C $\alpha$; OAS, oligo (A) synthetase; TRIM, tripartite motif; PKR, protein kinase R.

(Hsiang et al., 2012) (Figure 4A). NS1 is SUMOylated at residues K70 and K219. A non-SUMOylatable form of NS1 failed to inhibit IFN expression, and an excessive of NS1 SUMOylation also exerted a negative effect on its IFN-blocking function. Therefore, it is likely that an optimal level of NS1 SUMOylation is needed to endow NS1 with maximal activity of blocking IFN (Xu et al., 2011; Santos et al., 2013). ISGylation also limits influenza virus virulence. The IFN-induced ISG15 conjugation system can exert antiviral activity by conjugating ISG15 to NS1 in infected cells, thereby antagonizing various functions of NS1 and hence virus replication. ISG15 modification of NS1 K41 by the host E3 ligase Herc5 contributes to antagonizing IFN- $\beta$ response and disrupting the interaction of the NS1 RNA-binding domain (RBD) with importin- $\alpha$ (Zhao et al., 2010). Furthermore, Herc5-mediated ISGylation of NS1 K126 and K217 inhibits NS1 homodimerization, prevents RNA sequestration, impairs NS1 interaction with PKR, and interferes with the inhibitory effect of NS1 on virus-induced IFN- $\beta$ expression (Tang et al., 2010) (Figure 4D).

Influenza A viruses infection can activate the NLRP3 inflammasome, resulting in the production of IL-1 $\beta$, a major mediator of inflammation (Guo et al., 2015). However, this NLRP3 inflammasome-mediated IL-1 $\beta$ expression can be hindered by the $\mathrm{C}$ terminus of pandemic influenza virus NS1, which inhibits speck formation and ubiquitination of the inflammasome adaptor ASC (Park et al., 2018). Moreover, the ubiquitination sites $\mathrm{K} 110$ and $\mathrm{K} 140$ in the $\mathrm{C}$ terminus of porcine ASC are critical for both ASC ubiquitination and subsequent NLRP3 inflammasome-mediated IL-1 $\beta$ production (Park et al., 2018). Furthermore, NS1 counteracts the host NF-кB-mediated antiviral response by disrupting IKK function (Gao et al., 2012). More specifically, through its interaction with $\mathrm{IKK} \alpha / \mathrm{IKK} \beta$, NS1 not only blocks IKK $\beta$-mediated $\operatorname{IkB} \alpha$ phosphorylation and degradation in the cytoplasm but also impairs IKK-mediated histone $\mathrm{H} 3$ phosphorylation, which are crucial for triggering the rapid expression of NF- $\mathrm{B}$-regulated genes in the nucleus (Gao et al., 2012).

\section{Role of PTMs in Viral Resistance to Host IFN Responses Mediated by Other IAV Proteins}

The antiviral functions of other influenza virus proteins, including HA, PA, PA-X, and PB1-F2, are also regulated by PTMs. HA promotes the phosphorylation and polyubiquitination of IFNAR1, leading to an efficient degradation of IFNAR1 and attenuation of the type I IFN-induced antiviral signaling pathway (Xia et al., 2016). Furthermore, HA induces the degradation of type II IFN receptor 1 (IFNGR1), as well as of IFNAR1, resulting in the impairment of the cellular response to both types I and II IFNs (Xia et al., 2018). Mechanistically, the phosphorylation and ubiquitination of IFNGR1 and IFNAR1 mediated by the cellular 
kinase CK1 $\alpha$ contribute to the HA-induced elimination of type I and type II IFN receptors (Xia et al., 2018).

Polymerase acidic protein can block the activation of IFN- $\beta$ signaling pathway through the interaction with IRF3 (Yi et al., 2017). Mechanistically, through the endonuclease activity of its $\mathrm{N}$ terminus, PA interacts with IRF3 to suppress its phosphorylation, dimerization, and subsequent nuclear translocation and activation (Yi et al., 2017). The accessory proteins $\mathrm{PA}-\mathrm{X}$ and $\mathrm{PB} 1-\mathrm{F} 2$ also play a role in regulating the host innate immune response. PA-X is a fusion protein that incorporates the $\mathrm{N}$-terminal 191 amino acids PA with a short C-terminal sequence (either 61 or 41 codons) encoded by an overlapping open reading frame (ORF; "X-ORF") in segment 3 that is accessed by +1 ribosomal frameshifting (Jagger et al., 2012). This small protein acts to decrease the virulence of various influenza viruses through modulating the host innate immune response mainly through its host shutoff activity (Hu et al., 2018; Levene and Gaglia, 2018). Mechanistically, the NatB-mediated $\mathrm{N}$-terminal acetylation of PA-X contributes to this host shutoff activity and viral polymerase activity (Oishi et al., 2018). PB1F2, another accessory protein, is a 90 -amino acid protein that is encoded as an internal open reading frame on the PB1 gene of some influenza viruses. It contributes to the pathogenesis and comorbidity of influenza viruses mainly through manipulating apoptosis and innate immune responses, and enhancing the secondary bacterial infection (Cheung et al., 2020). Loss of PB1F2 ubiquitination enhances PB1-F2 stability, polymerase activity, and IFN antagonism (Kosik et al., 2015).

\section{Direct PTMs on Host Innate Immune-Related Proteins}

In addition to modification of influenza virus proteins, modification of host proteins also acts as a major mechanism of the host cellular response to control influenza virus infection. Various PTMs are directly involved in regulating the function of several crucial innate immune system proteins, such as IFITM3, TRIM28 (also known as KAP1), MAVS, and NEMO.

IFITM3 is a host antiviral restriction factor that limits cellular infection with multiple notable viral pathogens and is especially crucial for the innate immune response against influenza virus (Yount et al., 2010; Huang et al., 2011). The antiviral activity of IFITM3 is post-translationally regulated by various PTMs, particularly $S$-palmitoylation, ubiquitination, and monomethylation. S-palmitoylation controls IFITM3 clustering in membranes and contributes to its full antiviral activity against influenza virus (Yount et al., 2010). Ubiquitination of IFITM3 by the E3 ubiquitin ligase NEDD4 results in decreased IFITM3 expression and enhanced influenza viral infection (Chesarino et al., 2015). Additionally, IFITM3 K88 can be monomethylated (IFITM3-K88me1) by lysine methyltransferase SET7, resulting in a reduction in IFITM3 antiviral activity. To support efficient infection, IAV upregulates IFITM3 K88me1 by promoting the interaction of IFITM3 with SET7 (Shan et al., 2013). Interestingly, the ubiquitination of IFITM3 K88 can also inhibit IFITM3 antiviral activity. Therefore, the inhibition of these two negative regulatory modifications is speculated to increase antiviral activity (Yount et al., 2012). In contrast, the histone demethylase LSD1 can limit replication of RNA viruses through demethylating and activating IFITM3. However, influenza virus can inactivate IFITM3 by triggering IFITM3 methylation via promoting its disassociation from LSD1 (Shan et al., 2017).

TRIM28 has recently been implicated in contributing to inflammatory cytokine production during influenza virus infection (Domingues et al., 2015; Krischuns et al., 2018; Schmidt et al., 2019). Notably, the loss of SUMOylated TRIM28 results in an increased IFN-mediated antiviral response through the canonical components of dsRNA-sensing pathways, such as RIGI, MAVS, TBK1, and JAK-STAT (Domingues et al., 2015; Schmidt et al., 2019). Phosphorylation of TRIM28 S473 leads to enhanced expression levels of IFN- $\beta$, IL-6, and IL-8 during infection with highly pathogenic avian influenza viruses, such as H7N7, H7N9, and H5N1 (Krischuns et al., 2018).

Mitochondrial antiviral-signaling protein, a key mediator of IFN signaling, plays a critical role in host innate immunity against RNA viruses. PTMs of MAVS, such as K63-linked ubiquitination or phosphorylation, play a critical role in activating IFN signaling (Liu et al., 2015; Liu B. et al., 2017). Moreover, O-GlcNAcylation of MAVS by D-glucosamine was demonstrated to be required for influenza virus-induced MAVS K63-linked ubiquitination and the facilitation of subsequent IRF3 activation and IFN- $\beta$ production (Song et al., 2019).

NF-Kappa-B essential modulator is a crucial regulatory adaptor that is involved in both the IRF-mediated type I-IFN production signaling pathway and NF- $\kappa B$-mediated proinflammatory pathway. This protein can be ubiquitinated by the E3 ubiquitin ligase TRIM29 at K183 via a K48 linkage, leading to the inhibition of IRF signaling (Xing et al., 2016).

\section{ROLE OF PTMS IN INFLUENZA VIRUS-INDUCED CELL DEATH}

To subvert the host response, influenza virus induces early cell death of host cells through the manipulation of PTMs on the viral proteins PB1-F2, M2, and NS1 as well as on the innate immune factor ZBP1. PB1-F2 contains multiple PKC $\alpha$ phosphorylation sites, including S35 and T27. Functional analyses revealed that these two PB1-F2 phosphorylation sites play an important role in influenza virus replication and promote cellular apoptosis in primary human monocytes (Mitzner et al., 2009). M2 interacts closely with HSP40 and p58 (IPK) to form a stable complex, which leads to PKR autophosphorylation and activation, consequently inducing cell death (Guan et al., 2010). Moreover, the H7N9 influenza virus NS1 upregulated p53 expression through enhancing the phosphorylation levels of p53 and facilitating mitochondrial dysfunction, which may initiate NS1-induced apoptosis in human A549 cells (Yan et al., 2016). ZBP1 is an innate sensor of IAV infection (Kuriakose et al., 2016), and its activation contributes to IAV-induced cell death. Mechanistically, during influenza virus infection, RIGI-MAVS signaling, ZBP1 ubiquitination, and vRNP sensing by ZBP1 together contribute to the activation of $\mathrm{ZBP}$, 
resulting in the activation of programmed cell death pathways (Kesavardhana et al., 2017).

\section{ROLE OF PTMS IN INFLUENZA VIRUS HOST ADAPTATION}

During the process of influenza virus host adaptation to different species, the viruses develop different immune evasion strategies in different species. One study found that human TRIM25 bound to all the tested NS1, whereas chicken TRIM25 preferentially bound to avian NS1, and mouse TRIM25 was unable to bind to any of the NS1 (Rajsbaum et al., 2012) (Figure 3B). Accordingly, NS1 in human cells blocks RIG-I signaling by interacting with both human TRIM25 and human Riplet to inhibit RIG-I ubiquitination, whereas NS1 in mouse cells can suppress RIGI signaling only by binding to mouse Riplet (Rajsbaum et al., 2012) (Figures 3A,B), suggesting that species-specific strategies are used by NS1 to antagonize IFN production.

To replicate efficiently in humans, avian influenza viruses must adapt their viral RNA polymerase (vPol) to human cells. The nuclear proteins ANP32A and ANP32B function as cofactors for vPol activity (Sugiyama et al., 2015). Polymerase subunit PB2 627 is an important viral factor involved in influenza virus host adaption, and the E627K mutation in this subunit is an adaptive marker of avian influenza virus in mammalian hosts. A species-specific difference in ANP32A was reported to contribute to the host restriction of vPol. More specifically, the unique hydrophobic SIM-like sequence in avian ANP32A (avANP32A) enhanced the interaction of avANP32A with both human (PB2 627K) and avian-signature (PB2 627E) influenza virus vPols. However, human ANP32A (huANP32A) lacking the SIM-like sequence interacts weakly with both PB2 $627 \mathrm{~K}$ and PB2 627E vPols (Domingues and Hale, 2017). However, it was proposed that to replicate efficiently in human cells, PB2 627K vPol may increase the utilization efficiency of huANP32A rather than enhancing the binding activity of this protein (Domingues and Hale, 2017). Thus, for PB2 627E vPol to efficiently adapt to human cells, it must either overcome the weak huANP32A interaction or use huANP32A more efficiently.

The PTMs in HIST1H1C regulate the innate immunity as well as influenza virus replication. Specifically, the HIST1H1C phosphorylation mutant (containing T146A) has a weakened interaction with phosphorylated IRF3, which results in reduced IRF3 binding with the IFN- $\beta$ promoter and decreased IFN$\beta$ production. In contrast, HIST1H1C methylation mutants (containing K34A or K187A) have increased IFN- $\beta$ levels because they promote the binding of phosphorylated IRF3 to the IFN$\beta$ promoter (Liu X. K. et al., 2017). PB2 significantly affects HIST1H1C expression and modifications. Specifically, PB2 627K downregulates Sp1, and thereby decreases the binding of Sp1 to the HIST1H1C promoter, resulting in a reduction in HIST1H1C expression. HIST1H1C feedback then further downregulates DNA-PK and EHMT1/2 expression, consequently decreasing the phosphorylation and methylation levels of HIST1H1C (Liu X. et al., 2019). Therefore, PB2 627 regulates influenza virus host restriction via the regulation of HIST1H1C expression and modification.

\section{CONCLUDING REMARKS}

As an obligatory intracellular parasite, influenza virus heavily depends on host cellular factors and host cellular machineries to accomplish its life cycle and establish a successful infection. PTMs allow for the dynamic and reversible control of protein functions by modulating the protein abundance, interactors, catalysis, or localization. PTMs also act in many signaling pathways, such as those related to gene regulation, epigenetics, differentiation, protein degradation, tumorigenesis, and immune signaling. Furthermore, PTMs are important parts of the host immune response and play essential roles in replication for many viruses. For influenza virus, PTMs are involved in all steps of the viral replication cycle. The extensive usurpation of various host PTM-mediated pathways by influenza viruses at different stages of their life cycle emphasizes the crucial importance of these cellular machineries in cell physiology and function, and as a consequence, their importance for viral replication and viral diversion of cellular immunity. Currently, manipulation of the host PTMs systems is emerging as a key theme in terms of viral pathogenesis. Accumulating research has found clear evidence of viral proteins that mimic or redirect host PTMs to modify the cellular environment and influence the balance between normal and pathogenic cellular signaling in favor of virus persistence or efficient replication. However, given the wide sequence variation of the known candidates, it is a rather complicated task to systematically identify their viral analogs for ubiquitin or SUMO enzymes. In addition, proteins within released virions also contain multiple types of PTMs, although the function of these modifications within the virions is not currently well understood (Hutchinson et al., 2012).

The complexity of the interplay between viruses and the PTMs systems poses an exciting challenge for future research aiming to unravel the crosstalk between all of these modifications and assess the outcomes at a global level, which may shed further light on the complexity of influenza virus biology. Moreover, questions regarding whether all PTMs impact protein functions during virus infection or lead to dynamic changes in virus infection remain to be answered. There are also likely to be additional, as yet undiscovered, modes of post-translational regulation that occur on influenza virus proteins; their discovery will continue to add to our understanding of the role of PTMs systems during influenza virus infection. Additionally, the mechanisms of how viruses hijack the host PTMs machinery in the process of their coevolution with the host cells remain unknown. Moreover, considering the broad-ranging importance of PTMs in regulating viral replication and the host innate immune response to influenza viruses, more systematic research on this area should bring us closer to controlling and using PTMs for the development of preventative or antiviral therapeutic strategies and may also facilitate the 
study of innate immunity. Possible directions for future work could include clarifying the functional relevance of all PTMs involved in the specific stages of viral replication and systematically identifying the viral factors that interfere with PTMs.

In addition, given the growing appreciation for various PTMs and their biological functions during microorganism infection, the development of new approaches for studying PTMs is necessary. Presently, mass spectrometry can concurrently identify different types of PTMs, including phosphorylation, acetylation, methylation, and ubiquitination. Future work uncovering the dynamics of PTMs during microorganism infection will provide insight into the regulatory mechanisms and signaling pathways at a system-wide level. Top-down proteomics combined with time-resolved proteomics may reveal coincident modifications on proteins. Moreover, physiological approaches, such as the incorporation of unnatural amino acids with bioorthogonal reactivity (Neumann-Staubitz and Neumann, 2016), analog-sensitive AS kinases technology (Lopez et al., 2014), mass spectrometry imaging (Buchberger et al., 2018), and spatiotemporal visualization of PTMs using biosensors based on fluorescent proteins and fluorescence resonance energy transfer (Aye-Han et al., 2009; Hertel and Zhang, 2014) should also

\section{REFERENCES}

Altenberg, B., and Greulich, K. O. (2004). Genes of glycolysis are ubiquitously overexpressed in 24 cancer classes. Genomics 84, 1014-1020. doi: 10.1016/j. ygeno.2004.08.010

Arranz, R., Coloma, R., Chichon, F. J., Conesa, J. J., Carrascosa, J. L., Valpuesta, J. M., et al. (2012). The structure of native influenza virion ribonucleoproteins. Science 338, 1634-1637. doi: 10.1126/science.1228172

Aye-Han, N. N., Ni, Q., and Zhang, J. (2009). Fluorescent biosensors for real-time tracking of post-translational modification dynamics. Curr. Opin. Chem. Biol. 13, 392-397. doi: 10.1016/j.cbpa.2009.07.009

Banerjee, I., Miyake, Y., Nobs, S. P., Schneider, C., Horvath, P., Kopf, M., et al. (2014). Influenza A virus uses the aggresome processing machinery for host cell entry. Science 346, 473-477. doi: 10.1126/science.1257037

Barrett, T., Wolstenholme, A. J., and Mahy, B. W. (1979). Transcription and replication of influenza virus RNA. Virology 98, 211-225.

Buchberger, A. R., DeLaney, K., Johnson, J., and Li, L. (2018). Mass spectrometry imaging: a review of emerging advancements and future insights. Anal. Chem. 90, 240-265. doi: 10.1021/acs.analchem.7b04733

Bui, M., Wills, E. G., Helenius, A., and Whittaker, G. R. (2000). Role of the influenza virus M1 protein in nuclear export of viral ribonucleoproteins. J. Virol. 74, 1781-1786. doi: 10.1128/Jvi.74.4.1781-1786.2000

Calistri, A., Munegato, D., Carli, I., Parolin, C., and Palu, G. (2014). The ubiquitinconjugating system: multiple roles in viral replication and infection. Cells 3 , 386-417. doi: 10.3390/cells3020386

Chen, B. J., Takeda, M., and Lamb, R. A. (2005). Influenza virus hemagglutinin (H3 subtype) requires palmitoylation of its cytoplasmic tail for assembly: M1 proteins of two subtypes differ in their ability to support assembly. J. Virol. 79, 13673-13684. doi: 10.1128/JVI.79.21.13673-13684.2005

Chen, C., and Zhuang, X. (2008). Epsin 1 is a cargo-specific adaptor for the clathrin-mediated endocytosis of the influenza virus. Proc. Natl. Acad. Sci. U.S.A. 105, 11790-11795. doi: 10.1073/pnas.0803711105

Chen, L., Wang, C. M., Luo, J., Su, W., Li, M., Zhao, N., et al. (2017). Histone deacetylase 1 plays an acetylation-independent role in Influenza A virus replication. Front. Immunol. 8:1757. doi: 10.3389/Fimmu.2017.01757

Chesarino, N. M., McMichael, T. M., and Yount, J. S. (2015). E3 ubiquitin ligase NEDD4 promotes influenza virus infection by decreasing levels of the antiviral protein IFITM3. PLoS Pathog. 11:e1005095. doi: 10.1371/journal.ppat.1005095 accelerate our understanding the versatility of PTMs during virus infection.

\section{AUTHOR CONTRIBUTIONS}

$\mathrm{JH}$ and $\mathrm{XL}$ drafted and revised the manuscript. LZ contributed to the reference collection and analysis. All authors read and approved the final manuscript.

\section{FUNDING}

This work was supported by the National Key Research and Development Project of China (2016YFD0500202-1 and 2016YFD0501601), by the Special Financial Grant from the China Postdoctoral Science Foundation (2016T90515), by the earmarked fund for China Agriculture Research System (CARS40), by the "Qing Lan Project" of Higher Education Institutions of Jiangsu Province, China, by the "High-End Talent Support Program" of Yangzhou University, China, and by a project funded by the Priority Academic Program Development of Jiangsu Higher Education Institutions (PAPD).

Cheung, P. H., Lee, T. T., Chan, C. P., and Jin, D. Y. (2020). Influenza A virus PB1-F2 protein: an ambivalent innate immune modulator and virulence factor. J. Leukoc. Biol. 107, 763-771. doi: 10.1002/JLB.4MR0 320-206R

Cocucci, E., Aguet, F., Boulant, S., and Kirchhausen, T. (2012). The first five seconds in the life of a clathrin-coated pit. Cell 150, 495-507. doi: 10.1016/j. cell.2012.05.047

Cui, L., Mahesutihan, M., Zheng, W., Meng, L., Fan, W., Li, J., et al. (2018). CDC25B promotes influenza A virus replication by regulating the phosphorylation of nucleoprotein. Virology 525, 40-47. doi: 10.1016/j.virol. 2018.09.005

Daniels, R. S., Downie, J. C., Hay, A. J., Knossow, M., Skehel, J. J., Wang, M. L., et al. (1985). Fusion mutants of the influenza virus hemagglutinin glycoprotein. Cell 40, 431-439.

Dawson, A. R., and Mehle, A. (2018). Flu's cues: exploiting host post-translational modifications to direct the influenza virus replication cycle. PLoS Pathog. 14:e1007205. doi: 10.1371/journal.ppat.1007205

de Vries, E., Tscherne, D. M., Wienholts, M. J., Cobos-Jimenez, V., Scholte, F., Garcia-Sastre, A., et al. (2011). Dissection of the influenza A virus endocytic routes reveals macropinocytosis as an alternative entry pathway. PLoS Pathog. 7:e1001329. doi: 10.1371/journal.ppat.1001329

de Vries, R. P., de Vries, E., Bosch, B. J., de Groot, R. J., Rottier, P. J., and de Haan, C. A. (2010). The influenza A virus hemagglutinin glycosylation state affects receptor-binding specificity. Virology 403, 17-25. doi: 10.1016/j.virol.2010.03. 047

Deribe, Y. L., Pawson, T., and Dikic, I. (2010). Post-translational modifications in signal integration. Nat. Struct. Mol. Biol. 17, 666-672. doi: 10.1038/nsmb.1842

Di Pietro, A., Kajaste-Rudnitski, A., Oteiza, A., Nicora, L., Towers, G. J., Mechti, N., et al. (2013). TRIM22 inhibits influenza A virus infection by targeting the viral nucleoprotein for degradation. J. Virol. 87, 4523-4533. doi: 10.1128/JVI. 02548- 12

Domingues, P., Golebiowski, F., Tatham, M. H., Lopes, A. M., Taggart, A., Hay, R. T., et al. (2015). Global reprogramming of host SUMOylation during Influenza Virus Infection. Cell Rep. 13, 1467-1480. doi: 10.1016/j.celrep.2015. 10.001

Domingues, P., and Hale, B. G. (2017). Functional insights into ANP32Adependent Influenza A virus polymerase host restriction. Cell Rep. 20, 25382546. doi: 10.1016/j.celrep.2017.08.061 
Drazic, A., Myklebust, L. M., Ree, R., and Arnesen, T. (2016). The world of protein acetylation. Biochim. Biophys. Acta 1864, 1372-1401. doi: 10.1016/j.bbapap. 2016.06.007

Edinger, T. O., Pohl, M. O., and Stertz, S. (2014). Entry of influenza A virus: host factors and antiviral targets. J. Gen. Virol. 95(Pt 2), 263-277. doi: 10.1099/vir.0. 059477-0vir.0.059477-0

Engelhardt, O. G., Smith, M., and Fodor, E. (2005). Association of the influenza A virus RNA-dependent RNA polymerase with cellular RNA polymerase II. J. Virol. 79, 5812-5818. doi: 10.1128/JVI.79.9.5812-5818.2005

Esposito, V., Gargiulo, M., Parrella, R., Sangiovanni, V., Viglietti, R., Fortunato, V., et al. (2009). Role of Nedd8 in HIV-associated lipodystrophy. Infection 37:83.

Fu, B., Wang, L., Ding, H., Schwamborn, J. C., Li, S., and Dorf, M. E. (2015). TRIM32 senses and restricts Influenza A virus by ubiquitination of $\mathrm{PB} 1$ polymerase. PLoS Pathog. 11:e1004960. doi: 10.1371/journal.ppat.1004960

Gack, M. U., Albrecht, R. A., Urano, T., Inn, K. S., Huang, I. C., Carnero, E., et al. (2009). Influenza A virus NS1 targets the ubiquitin ligase TRIM25 to evade recognition by the host viral RNA sensor RIG-I. Cell Host Microbe 5, 439-449. doi: 10.1016/j.chom.2009.04.006

Gambaryan, A. S., Tuzikov, A. B., Pazynina, G. V., Desheva, J. A., Bovin, N. V., Matrosovich, M. N., et al. (2008). 6-sulfo sialyl Lewis X is the common receptor determinant recognized by H5, H6, H7 and H9 influenza viruses of terrestrial poultry. Virol. J. 5:85. doi: 10.1186/1743-422X-5-851743-422X-5-85

Gamblin, S. J., Haire, L. F., Russell, R. J., Stevens, D. J., Xiao, B., Ha, Y., et al. (2004). The structure and receptor binding properties of the 1918 influenza hemagglutinin. Science 303, 1838-1842. doi: 10.1126/science.10931551093155

Gao, S., Song, L., Li, J., Zhang, Z., Peng, H., Jiang, W., et al. (2012). Influenza A virus-encoded NS1 virulence factor protein inhibits innate immune response by targeting IKK. Cell Microbiol. 14, 1849-1866. doi: 10.1111/cmi.12005

Gao, S. J., Wu, J. X., Liu, R. Y., Li, J. D., Song, L. P., Teng, Y., et al. (2015). Interaction of NS2 with AIMP2 facilitates the switch from ubiquitination to SUMOylation of M1 in Influenza A virus-infected cells. J. Virol. 89, 300-311. doi: 10.1128/Jvi.02170- 14

Garcia-Sastre, A., Durbin, R. K., Zheng, H., Palese, P., Gertner, R., Levy, D. E., et al. (1998). The role of interferon in influenza virus tissue tropism. J. Virol. $72,8550-8558$.

Giese, S., Ciminski, K., Bolte, H., Moreira, E. A., Lakdawala, S., Hu, Z., et al. (2017). Role of influenza A virus NP acetylation on viral growth and replication. Nat. Commun. 8:1259. doi: 10.1038/s41467-017-01112-3

Guan, Z. H., Liu, D., Mi, S. F., Zhang, J., Ye, Q. N., Wang, M., et al. (2010). Interaction of Hsp40 with influenza virus M2 protein: implications for PKR signaling pathway. Protein Cell 1, 944-955. doi: 10.1007/s13238-010-0115-x

Guo, H. T., Callaway, J. B., and Ting, J. P. Y. (2015). Inflammasomes: mechanism of action, role in disease, and therapeutics. Nat. Med. 21, 677-687. doi: 10.1038/ nm.3893

Haidari, M., Zhang, W., Ganjehei, L., Ali, M., and Chen, Z. (2011). Inhibition of MLC phosphorylation restricts replication of influenza virus-a mechanism of action for anti-influenza agents. PLoS One 6:e21444. doi: 10.1371/journal.pone. 0021444

Hale, B. G., Knebel, A., Botting, C. H., Galloway, C. S., Precious, B. L., Jackson, D., et al. (2009). CDK/ERK-mediated phosphorylation of the human influenza A virus NS1 protein at threonine-215. Virology 383, 6-11. doi: 10.1016/j.virol. 2008.10.002

Hara, K., Shiota, M., Kido, H., Ohtsu, Y., Kashiwagi, T., Iwahashi, J., et al. (2001). Influenza virus RNA polymerase PA subunit is a novel serine protease with Ser624 at the active site. Genes Cells 6, 87-97. doi: 10.1046/j.1365-2443.2001. 00399.x

Hara, K., Shiota, M., Kido, H., Watanabe, K., Nagata, K., and Toyoda, T. (2003). Inhibition of the protease activity of influenza virus RNA polymerase PA subunit by viral matrix protein. Microbiol. Immunol. 47, 521-526. doi: 10.1111/ j.1348-0421.2003.tb03413.x

Hatakeyama, D., Shoji, M., Yamayoshi, S., Yoh, R., Ohmi, N., Takenaka, S., et al. (2018). Influenza A virus nucleoprotein is acetylated by histone acetyltransferases PCAF and GCN5. J. Biol. Chem. 293, 7126-7138. doi: 10. 1074/jbc.RA117.001683

Hay, A. J., Skehel, J. J., and McCauley, J. (1982). Characterization of influenza virus RNA complete transcripts. Virology 116, 517-522.
Herfst, S., Schrauwen, E. J., Linster, M., Chutinimitkul, S., de Wit, E., Munster, V. J., et al. (2012). Airborne transmission of influenza A/H5N1 virus between ferrets. Science 336, 1534-1541. doi: 10.1126/science. 1213362

Hertel, F., and Zhang, J. (2014). Monitoring of post-translational modification dynamics with genetically encoded fluorescent reporters. Biopolymers 101, 180-187. doi: 10.1002/bip.22254

Hirata, N., Suizu, F., Matsuda-Lennikov, M., Edamura, T., Bala, J., and Noguchi, M. (2014). Inhibition of Akt kinase activity suppresses entry and replication of influenza virus. Biochem. Biophys. Res. Commun. 450, 891-898. doi: 10.1016/j. bbrc.2014.06.077

Hsiang, T. Y., Zhou, L. G., and Krug, R. M. (2012). Roles of the phosphorylation of specific serines and threonines in the NS1 protein of human Influenza A viruses. J. Virol. 86, 10370-10376. doi: 10.1128/Jvi.00732-12

$\mathrm{Hu}, \mathrm{J} ., \mathrm{Ma}, \mathrm{C}$., and Liu, X. (2018). PA-X: a key regulator of influenza A virus pathogenicity and host immune responses. Med. Microbiol. Immunol. 207, 255-269. doi: 10.1007/s00430-018-0548-z

Huang, I. C., Bailey, C. C., Weyer, J. L., Radoshitzky, S. R., Becker, M. M., Chiang, J. J., et al. (2011). Distinct patterns of IFITM-mediated restriction of filoviruses, SARS coronavirus, and Influenza A Virus. PLoS Pathog. 7:e1001258. doi: 10. 1371/journal.ppat.1001258

Huang, R. T., Lichtenberg, B., and Rick, O. (1996). Involvement of annexin V in the entry of influenza viruses and role of phospholipids in infection. FEBS Lett. 392, 59-62. doi: 10.1016/0014-5793(96)00783-1

Hughes, D. J., Wood, J. J., Jackson, B. R., Baquero-Perez, B., and Whitehouse, A. (2015). NEDDylation is essential for kaposi's sarcoma- associated herpesvirus latency and lytic reactivation and represents a novel Anti-KSHV target. PLoS Pathog. 11:e1004771. doi: 10.1371/journal.ppat.1004771

Husain, M., and Cheung, C. Y. (2014). Histone deacetylase 6 inhibits influenza A virus release by downregulating the trafficking of viral components to the plasma membrane via its substrate, acetylated microtubules. J. Virol. 88, 11229-11239. doi: 10.1128/JVI.00727-14JVI.00727-14

Husain, M., and Harrod, K. S. (2011). Enhanced acetylation of alpha-tubulin in influenza A virus infected epithelial cells. FEBS Lett. 585, 128-132. doi: 10.1016/ j.febslet.2010.11.023

Hutchinson, E. C., Denham, E. M., Thomas, B., Trudgian, D. C., Hester, S. S., Ridlova, G., et al. (2012). Mapping the phosphoproteome of Influenza A and B viruses by mass spectrometry. PLoS Pathog. 8:e1002993. doi: 10.1371/journal. ppat.1002993

Hutchinson, E. C., and Fodor, E. (2013). Transport of the influenza virus genome from nucleus to nucleus. Viruses 5, 2424-2446. doi: 10.3390/v5102424v5102424

Iwasaki, A., and Pillai, P. S. (2014). Innate immunity to influenza virus infection. Nat. Rev. Immunol. 14, 315-328. doi: 10.1038/nri3665

Jagger, B. W., Wise, H. M., Kash, J. C., Walters, K. A., Wills, N. M., Xiao, Y. L., et al. (2012). An overlapping protein-coding region in influenza A virus segment 3 modulates the host response. Science 337, 199-204. doi: 10.1126/science. 1222213

Janke, C., and Bulinski, J. C. (2011). Post-translational regulation of the microtubule cytoskeleton: mechanisms and functions. Nat. Rev. Mol. Cell Biol. 12, 773-786. doi: 10.1038/nrm3227nrm3227

Jiang, W., Wang, Q., Chen, S., Gao, S., Song, L., Liu, P., et al. (2013). Influenza A virus NS1 induces G0/G1 cell cycle arrest by inhibiting the expression and activity of RhoA protein. J. Virol. 87, 3039-3052. doi: 10.1128/JVI.03176-12JVI. 03176-12

Johnson, E. S. (2004). Protein modification by SUMO. Annu. Rev. Biochem. 73, 355-382. doi: 10.1146/annurev.biochem.73.011303.074118

Kamata, T., and Watanabe, Y. (1977). Role for nucleocapsid protein phosphorylation in the transcription of influenza virus genome. Nature 267, 460-462. doi: 10.1038/267460a0

Kathum, O. A., Schrader, T., Anhlan, D., Nordhoff, C., Liedmann, S., Pande, A., et al. (2016). Phosphorylation of influenza A virus NS1 protein at threonine 49 suppresses its interferon antagonistic activity. Cell. Microbiol. 18, 784-791. doi: $10.1111 / \mathrm{cmi} .12559$

Kesavardhana, S., Kuriakose, T., Guy, C. S., Samir, P., Malireddi, R. K. S., Mishra, A., et al. (2017). ZBP1/DAI ubiquitination and sensing of influenza vRNPs activate programmed cell death. J. Exp. Med. 214, 2217-2229. doi: 10.1084/jem. 20170550 
Khor, R., McElroy, L. J., and Whittaker, G. R. (2003). The ubiquitin-vacuolar protein sorting system is selectively required during entry of influenza virus into host cells. Traffic 4, 857-868. doi: 10.1046/j.1600-0854.2003.00140.x

Kirui, J., Mondal, A., and Mehle, A. (2016). Ubiquitination upregulates influenza virus polymerase function. J. Virol. 90, 10906-10914. doi: 10.1128/JVI.0182916

Koliopoulos, M. G., Lethier, M., van der Veen, A. G., Haubrich, K., Hennig, J., Kowalinski, E., et al. (2018). Molecular mechanism of influenza A NS1mediated TRIM25 recognition and inhibition. Nat. Commun. 9:1820. doi: 10. 1038/s41467-018-04214-8

Konig, R., Stertz, S., Zhou, Y., Inoue, A., Hoffmann, H. H., Bhattacharyya, S., et al. (2010). Human host factors required for influenza virus replication. Nature 463, 813-817. doi: 10.1038/nature08699

Kosik, I., Praznovska, M., Kosikova, M., Bobisova, Z., Holly, J., Vareckova, E., et al. (2015). The ubiquitination of the influenza A virus PB1-F2 protein is crucial for its biological function. PLoS One 10:e0118477. doi: 10.1371/journal.pone. 0118477

Krischuns, T., Gunl, F., Henschel, L., Binder, M., Willemsen, J., Schloer, S., et al. (2018). Phosphorylation of TRIM28 enhances the expression of IFN-beta and proinflammatory cytokines during HPAIV infection of human lung epithelial cells. Front. Immunol. 9:2229. doi: 10.3389/fimmu.2018.02229

Kuriakose, T., Man, S. M., Malireddi, R. K. S., Karki, R., Kesavardhana, S., Place, D. E., et al. (2016). ZBP1/DAI is an innate sensor of influenza virus triggering the NLRP3 inflammasome and programmed cell death pathways. Sci. Immunol. 1:aag2045. doi: 10.1126/sciimmunol.aag2045

Kuss-Duerkop, S. K., Wang, J., Mena, I., White, K., Metreveli, G., Sakthivel, R., et al. (2017). Influenza virus differentially activates mTORC1 and mTORC2 signaling to maximize late stage replication. PLoS Pathog. 13:e1006635. doi: 10.1371/journal.ppat.1006635

Lakdawala, S. S., Fodor, E., and Subbarao, K. (2016). Moving on out: transport and packaging of influenza viral RNA into virions. Annu. Rev. Virol. 3, 411-427. doi: 10.1146/annurev-virology-110615-142345

Levene, R. E., and Gaglia, M. M. (2018). Host shutoff in Influenza A virus: many means to an end. Viruses 10:475. doi: 10.3390/v10090475

Li, Y., Sun, L., Zheng, W. N., Mahesutihan, M., Li, J., Bi, Y. H., et al. (2018). Phosphorylation and dephosphorylation of threonine 188 in nucleoprotein is crucial for the replication of influenza A virus. Virology 520, 30-38. doi: 10 . 1016/j.virol.2018.05.002

Liao, T. L., Wu, C. Y., Su, W. C., Jeng, K. S., and Lai, M. M. (2010). Ubiquitination and deubiquitination of NP protein regulates influenza A virus RNA replication. EMBO J. 29, 3879-3890. doi: 10.1038/emboj.2010.250

Lin, Y. C., Jeng, K. S., and Lai, M. M. C. (2017). CNOT4-mediated ubiquitination of Influenza A virus nucleoprotein promotes viral RNA replication. mBio mBio:e00597-17. doi: 10.1128/mBio.00597-17

Lincoln, T. M. (2007). Myosin phosphatase regulatory pathways: different functions or redundant functions? Circ. Res. 100, 10-12. doi: 10.1161/01.RES. 0000255894.25293 .82

Liu, B., Zhang, M., Chu, H., Zhang, H., Wu, H., Song, G., et al. (2017). The ubiquitin E3 ligase TRIM31 promotes aggregation and activation of the signaling adaptor MAVS through Lys63-linked polyubiquitination. Nat. Immunol. 18, 214-224. doi: $10.1038 /$ ni. 3641

Liu, X. K., Yang, C., Hu, Y., Lei, E. M., Lin, X., Zhao, L. Z., et al. (2017). HIST1H1C regulates interferon-beta and inhibits influenza virus replication by interacting with IRF3. Front. Immunol. 8:350. doi: 10.3389/Fimmu.2017. 00350

Liu, S., Cai, X., Wu, J., Cong, Q., Chen, X., Li, T., et al. (2015). Phosphorylation of innate immune adaptor proteins MAVS, STING, and TRIF induces IRF3 activation. Science 347:aaa2630. doi: 10.1126/science.aaa2630

Liu, S., Liu, L., Xu, G., Cao, Z. Y., Wang, Q., Li, S., et al. (2019). Epigenetic modification is regulated by the interaction of Influenza A virus nonstructural protein 1 with the de novo DNA methyltransferase DNMT3B and subsequent transport to the cytoplasm for K48-linked polyubiquitination. J. Virol. 93:e01587-18. doi: 10.1128/JVI.01587-18

Liu, X., Yang, C., Sun, X., Lin, X., Zhao, L., Chen, H., et al. (2019). Evidence for a novel mechanism of influenza A virus host adaptation modulated by PB2-627. FEBS J. 286, 3389-3400. doi: 10.1111/febs.14867

Londrigan, S. L., Turville, S. G., Tate, M. D., Deng, Y. M., Brooks, A. G., and Reading, P. C. (2011). N-linked glycosylation facilitates sialic acid-independent attachment and entry of influenza A viruses into cells expressing DC-SIGN or L-SIGN. J. Virol. 85, 2990-3000. doi: 10.1128/JVI.01705-10JVI.01705-10

Lopez, M. S., Kliegman, J. I., and Shokat, K. M. (2014). The logic and design of analog-sensitive kinases and their small molecule inhibitors. Methods Enzymol. 548, 189-213. doi: 10.1016/B978-0-12-397918-6.00008-2

Maeda, T., and Ohnishi, S. (1980). Activation of influenza virus by acidic media causes hemolysis and fusion of erythrocytes. FEBS Lett. 122, 283-287. doi: 10.1016/0014-5793(80)80457-1

Mahmoudian, S., Auerochs, S., Grone, M., and Marschall, M. (2009). Influenza A virus proteins $\mathrm{PB} 1$ and NS1 are subject to functionally important phosphorylation by protein kinase C. J. Gen. Virol. 90(Pt 6), 1392-1397. doi: 10.1099/vir.0.009050-0

Marazzi, I., Ho, J. S., Kim, J., Manicassamy, B., Dewell, S., Albrecht, R. A., et al. (2012). Suppression of the antiviral response by an influenza histone mimic. Nature 483, 428-433. doi: 10.1038/nature10892

Marjuki, H., Alam, M. I., Ehrhardt, C., Wagner, R., Planz, O., Klenk, H. D., et al. (2006). Membrane accumulation of influenza A virus hemagglutinin triggers nuclear export of the viral genome via protein kinase Calpha-mediated activation of ERK signaling. J. Biol. Chem. 281, 16707-16715. doi: 10.1074/jbc. M510233200

Martin, K., and Helenius, A. (1991). Transport of incoming influenza virus nucleocapsids into the nucleus. J. Virol. 65, 232-244.

Mata, M. A., Satterly, N., Versteeg, G. A., Frantz, D., Wei, S. G., Williams, N., et al. (2011). Chemical inhibition of RNA viruses reveals REDD1 as a host defense factor. Nat. Chem. Biol. 7, 712-719. doi: 10.1038/Nchembio.645

Matlin, K. S., Reggio, H., Helenius, A., and Simons, K. (1981). Infectious entry pathway of influenza virus in a canine kidney cell line. J. Cell Biol. 91(3 Pt 1), 601-613. doi: 10.1083/jcb.91.3.601

Mitzner, D., Dudek, S. E., Studtrucker, N., Anhlan, D., Mazur, I., Wissing, J., et al. (2009). Phosphorylation of the influenza A virus protein PB1-F2 by PKC is crucial for apoptosis promoting functions in monocytes. Cell. Microbiol. 11, 1502-1516. doi: 10.1111/j.1462-5822.2009.01343.x

Miyake, Y., Ishii, K., and Honda, A. (2017). Influenza virus infection induces host pyruvate kinase $\mathrm{M}$ which interacts with viral RNA-dependent RNA polymerase. Front. Microbiol. 8:162. doi: 10.3389/Fmicb.2017.00162

Moeller, A., Kirchdoerfer, R. N., Potter, C. S., Carragher, B., and Wilson, I. A. (2012). Organization of the influenza virus replication machinery. Science 338, 1631-1634. doi: 10.1126/science. 1227270

Mondal, A., Dawson, A. R., Potts, G. K., Freiberger, E. C., Baker, S. F., Moser, L. A., et al. (2017). Influenza virus recruits host protein kinase C to control assembly and activity of its replication machinery. eLife 6:e26910. doi: 10.7554/ eLife. 26910

Mondal, A., Potts, G. K., Dawson, A. R., Coon, J. J., and Mehle, A. (2015). Phosphorylation at the homotypic interface regulates nucleoprotein oligomerization and assembly of the influenza virus replication machinery. PLoS Pathog. 11:e1004826. doi: 10.1371/journal.ppat.1004826

Neumann, G., Castrucci, M. R., and Kawaoka, Y. (1997). Nuclear import and export of influenza virus nucleoprotein. J. Virol. 71, 9690-9700.

Neumann-Staubitz, P., and Neumann, H. (2016). The use of unnatural amino acids to study and engineer protein function. Curr. Opin. Struct. Biol. 38, 119-128. doi: 10.1016/j.sbi.2016.06.006

Nogales, A., Martinez-Sobrido, L., Topham, D. J., and DeDiego, M. L. (2018). Modulation of innate immune responses by the Influenza A NS1 and PA-X Proteins. Viruses 10:708. doi: 10.3390/v10120708

Nunes-Correia, I., Eulalio, A., Nir, S., Pedroso, and de Lima, M. C. (2004). Caveolae as an additional route for influenza virus endocytosis in MDCK cells. Cell Mol. Biol. Lett. 9, 47-60.

Oishi, K., Yamayoshi, S., Kozuka-Hata, H., Oyama, M., and Kawaoka, Y. (2018). $\mathrm{N}$-Terminal acetylation by NatB is required for the shutoff activity of Influenza A virus PA-X. Cell Rep. 24, 851-860. doi: 10.1016/j.celrep.2018.06.078

O’Neill, R. E., Jaskunas, R., Blobel, G., Palese, P., and Moroianu, J. (1995). Nuclear import of influenza virus RNA can be mediated by viral nucleoprotein and transport factors required for protein import. J. Biol. Chem. 270, 22701-22704. doi: $10.1074 /$ jbc. 270.39 .22701

Panella, S., Marcocci, M. E., Celestino, I., Valente, S., Zwergel, C., Puma, D. D. L., et al. (2016). MC1568 inhibits HDAC6/8 activity and influenza A virus replication in lung epithelial cells: role of Hsp90 acetylation. Fut. Med. Chem. 8, 2017-2031. doi: 10.4155/fmc-2016-73 
Park, H. S., Liu, G. Q., Raman, S. N. T., Landreth, S. L., Liu, Q., and Zhou, Y. (2018). NS1 protein of 2009 pandemic Influenza A virus inhibits porcine NLRP3 inflammasome-mediated Interleukin-1 beta production by suppressing ASC ubiquitination. J. Virol. 92:e00022-18. doi: 10.1128/JVI.00022-18

Paterson, D., and Fodor, E. (2012). Emerging roles for the influenza A virus nuclear export protein (NEP). PLoS Pathog. 8:e1003019. doi: 10.1371/journal. ppat.1003019

Patil, G., Zhao, M., Song, K., Hao, W., Bouchereau, D., Wang, L., et al. (2018). TRIM41-mediated ubiquitination of nucleoprotein limits Influenza A virus infection. J. Virol. 92:e0905-18. doi: 10.1128/JVI.00905-18

Perales, B., Sanz-Ezquerro, J. J., Gastaminza, P., Ortega, J., Santaren, J. F., Ortin, J., et al. (2000). The replication activity of influenza virus polymerase is linked to the capacity of the PA subunit to induce proteolysis. J. Virol. 74, 1307-1312. doi: 10.1128/Jvi.74.3.1307-1312.2000

Pickart, C. M. (2004). Back to the future with ubiquitin. Cell 116, 181-190. doi: 10.1016/s0092-8674(03)01074-2

Pleschka, S., Wolff, T., Ehrhardt, C., Hobom, G., Planz, O., Rapp, U. R., et al. (2001). Influenza virus propagation is impaired by inhibition of the Raf/MEK/ERK signalling cascade. Nat. Cell Biol. 3, 301-305. doi: 10.1038/35060098

Plotch, S. J., Bouloy, M., Ulmanen, I., and Krug, R. M. (1981). A unique cap $(m 7 G p p p X m)$-dependent influenza virion endonuclease cleaves capped RNAs to generate the primers that initiate viral RNA transcription. Cell 23, 847-858. doi: 10.1016/0092-8674(81)90449-9

Predicala, R., and Zhou, Y. (2013). The role of Ran-binding protein 3 during influenza A virus replication. J. Gen. Virol. 94(Pt 5), 977-984. doi: 10.1099/vir. 0.049395-0

Qian, W., Wei, X. Q., Guo, K. L., Li, Y. T., Lin, X., Zou, Z., et al. (2017). The c-Terminal effector domain of non-structural protein 1 of influenza a virus blocks IFN-beta production by targeting TnF receptor-associated Factor 3. Front. Immunol. 8:779. doi: 10.3389/Fimmu.2017.00779

Radtke, K., Dohner, K., and Sodeik, B. (2006). Viral interactions with the cytoskeleton: a hitchhiker's guide to the cell. Cell. Microbiol. 8, 387-400. doi: $10.1111 / \mathrm{j} .1462-5822.2005 .00679 . \mathrm{x}$

Rajsbaum, R., Albrecht, R. A., Wang, M. K., Maharaj, N. P., Versteeg, G. A., NistalVillan, E., et al. (2012). Species-specific inhibition of RIG-I ubiquitination and IFN induction by the influenza A virus NS1 protein. PLoS Pathog. 8:e1003059. doi: 10.1371/journal.ppat.1003059

Roy, A. M., Parker, J. S., Parrish, C. R., and Whittaker, G. R. (2000). Early stages of influenza virus entry into Mv-1 lung cells: involvement of dynamin. Virology 267, 17-28. doi: 10.1006/viro.1999.0109

Rudnicka, A., and Yamauchi, Y. (2016). Ubiquitin in influenza virus entry and innate immunity. Viruses 8:293. doi: 10.3390/v8100293

Rust, M. J., Lakadamyali, M., Zhang, F., and Zhuang, X. (2004). Assembly of endocytic machinery around individual influenza viruses during viral entry. Nat. Struct. Mol. Biol. 11, 567-573. doi: 10.1038/nsmb769

Santos, A., Pal, S., Chacon, J., Meraz, K., Gonzalez, J., Prieto, K., et al. (2013). SUMOylation affects the interferon blocking activity of the influenza A nonstructural protein NS1 without affecting its stability or cellular localization. J. Virol. 87, 5602-5620. doi: 10.1128/JVI.02063-12

Schmidt, N., Domingues, P., Golebiowski, F., Patzina, C., Tatham, M. H., Hay, R. T., et al. (2019). An influenza virus-triggered SUMO switch orchestrates co-opted endogenous retroviruses to stimulate host antiviral immunity. Proc. Natl. Acad. Sci. U.S.A. 116, 17399-17408. doi: 10.1073/pnas.1907031116

Schmitt, A. P., and Lamb, R. A. (2005). Influenza virus assembly and budding at the viral budozone. Virus Struct. Assem. 64, 383-416. doi: 10.1016/S0065-3527(05) 64012-2

Shan, J., Zhao, B., Shan, Z., Nie, J., Deng, R., Xiong, R., et al. (2017). Histone demethylase LSD1 restricts influenza A virus infection by erasing IFITM3K88 monomethylation. PLoS Pathog. 13:e1006773. doi: 10.1371/journal.ppat. 1006773

Shan, Z., Han, Q., Nie, J., Cao, X., Chen, Z., Yin, S., et al. (2013). Negative regulation of interferon-induced transmembrane protein 3 by SET7-mediated lysine monomethylation. J. Biol. Chem. 288, 35093-35103. doi: 10.1074/jbc. M113.511949

Sieczkarski, S. B., and Whittaker, G. R. (2002). Influenza virus can enter and infect cells in the absence of clathrin-mediated endocytosis. J. Virol. 76, 10455-10464. doi: 10.1128/jvi.76.20.10455-10464.2002
Song, N., Qi, Q., Cao, R., Qin, B., Wang, B., Wang, Y., et al. (2019). MAVS O-GlcNAcylation is essential for host antiviral immunity against lethal RNA viruses. Cell Rep. 28, 2386.e5-2396.e5. doi: 10.1016/j.celrep.2019.07.085

Soucy, T. A., Dick, L. R., Smith, P. G., Milhollen, M. A., and Brownell, J. E. (2010). The NEDD8 conjugation pathway and its relevance in cancer biology and therapy. Genes Cancer 1, 708-716. doi: 10.1177/1947601910382898

Stanley, D. J., Bartholomeeusen, K., Crosby, D. C., Kim, D. Y., Kwon, E., Yen, L., et al. (2012). Inhibition of a NEDD8 cascade restores restriction of HIV by APOBEC3G. PLoS Pathog. 8:e1003085. doi: 10.1371/journal.ppat.1003085

Stevens, J., Blixt, O., Tumpey, T. M., Taubenberger, J. K., Paulson, J. C., and Wilson, I. A. (2006). Structure and receptor specificity of the hemagglutinin from an H5N1 influenza virus. Science 312, 404-410. doi: 10.1126/science.1124513

Su, W. C., Chen, Y. C., Tseng, C. H., Hsu, P. W. C., Tung, K. F., Jeng, K. S., et al. (2013). Pooled RNAi screen identifies ubiquitin ligase Itch as crucial for influenza A virus release from the endosome during virus entry. Proc. Natl. Acad. Sci. U.S.A. 110, 17516-17521. doi: 10.1073/pnas.1312374110

Su, W. C., Yu, W. Y., Huang, S. H., and Lai, M. M. C. (2018). Ubiquitination of the cytoplasmic domain of Influenza A virus M2 protein is crucial for production of infectious virus particles. J. Virol. 92:e01972-17. doi: 10.1128/JVI.01972-17

Sugiyama, K., Kawaguchi, A., Okuwaki, M., and Nagata, K. (2015). pp32 and APRIL are host cell-derived regulators of influenza virus RNA synthesis from cRNA. eLife 4:e08939. doi: 10.7554/eLife.08939

Tan, B., and Gao, S. J. (2018). RNA epitranscriptomics: regulation of infection of RNA and DNA viruses by N(6) -methyladenosine (m(6) A). Rev. Med. Virol. 28:e1983. doi: $10.1002 /$ rmv. 1983

Tang, Y., Zhong, G., Zhu, L., Liu, X., Shan, Y., Feng, H., et al. (2010). Herc5 attenuates influenza A virus by catalyzing ISGylation of viral NS1 protein. J. Immunol. 184, 5777-5790. doi: 10.4049/jimmunol.0903588

Tao, Y. J., and Ye, Q. Z. (2010). RNA virus replication complexes. PLoS Pathog. 6:e1000943. doi: 10.1371/journal.ppat.1000943

Turrell, L., Hutchinson, E. C., Vreede, F. T., and Fodor, E. (2015). Regulation of influenza A virus nucleoprotein oligomerization by phosphorylation. J. Virol. 89, 1452-1455. doi: 10.1128/JVI.02332-14

Vreede, F. T., Jung, T. E., and Brownlee, G. G. (2004). Model suggesting that replication of influenza virus is regulated by stabilization of replicative intermediates. J. Virol. 78, 9568-9572. doi: 10.1128/Jvi.78.17.9568-9572.2004

Wang, S. S., Zhao, Z. D., Bi, Y. H., Sun, L., Liu, X. L., and Liu, W. J. (2013). Tyrosine 132 phosphorylation of Influenza A virus M1 protein is crucial for virus replication by controlling the nuclear import of M1. J. Virol. 87, 6182-6191. doi: 10.1128/Jvi.03024-012

Weis, W., Brown, J. H., Cusack, S., Paulson, J. C., Skehel, J. J., and Wiley, D. C. (1988). Structure of the influenza virus haemagglutinin complexed with its receptor, sialic acid. Nature 333, 426-431. doi: 10.1038/333426a0

Welchman, R. L., Gordon, C., and Mayer, R. J. (2005). Ubiquitin and ubiquitinlike proteins as multifunctional signals. Nat. Rev. Mol. Cell Biol. 6, 599-609. doi: $10.1038 / \mathrm{nrm} 1700$

White, J. M., and Wilson, I. A. (1987). Anti-peptide antibodies detect steps in a protein conformational change: low-pH activation of the influenza virus hemagglutinin. J. Cell Biol. 105(6 Pt 2), 2887-2896. doi: 10.1083/jcb.105.6.2887

Widjaja, I., de Vries, E., Tscherne, D. M., Garcia-Sastre, A., Rottier, P. J. M., and de Haan, C. A. M. (2010). Inhibition of the ubiquitin-proteasome system affects Influenza A virus infection at a postfusion step. J. Virol. 84, 9625-9631. doi: 10.1128/Jvi.01048-10

Winkler, R., Gillis, E., Lasman, L., Safra, M., Geula, S., Soyris, C., et al. (2019). $\mathrm{m}(6) \mathrm{A}$ modification controls the innate immune response to infection by targeting type I interferons. Nat. Immunol. 20, 173-182. doi: 10.1038/s41590018-0275-Z

Wu, C. Y., Jeng, K. S., and Lai, M. M. C. (2011). The SUMOylation of matrix protein M1 modulates the assembly and morphogenesis of Influenza A virus. J. Virol. 85, 6618-6628. doi: 10.1128/Jvi.02401-10

Wu, X., Wang, J., Wang, S., Wu, F., Chen, Z., Li, C., et al. (2019). Inhibition of Influenza A virus replication by TRIM14 via its multifaceted protein-protein interaction With NP. Front. Microbiol. 10:344. doi: 10.3389/fmicb.2019.00344

Xia, C., Vijayan, M., Pritzl, C. J., Fuchs, S. Y., McDermott, A. B., and Hahm, B. (2016). Hemagglutinin of Influenza A virus antagonizes Type I Interferon (IFN) responses by inducing degradation of Type I IFN receptor 1. J. Virol. 90, 2403-2417. doi: 10.1128/Jvi.02749-15 
Xia, C., Wolf, J. J., Vijayan, M., Studstill, C. J., Ma, W., and Hahm, B. (2018). Casein kinase 1alpha mediates the degradation of receptors for Type I and Type II interferons caused by hemagglutinin of Influenza A virus. J. Virol. 92:e00006-18. doi: 10.1128/JVI.00006-18

Xing, J. J., Weng, L. Y., Yuan, B., Wang, Z., Jia, L., Jin, R., et al. (2016). Identification of a role for TRIM29 in the control of innate immunity in the respiratory tract (vol 17, pg 1373, 2016). Nat. Immunol. 17, 1479-1479. doi: 10.1038/Ni1216$1479 \mathrm{a}$

Xu, K., Klenk, C., Liu, B., Keiner, B., Cheng, J., Zheng, B. J., et al. (2011). Modification of nonstructural protein 1 of influenza A virus by SUMO1. J. Virol. 85, 1086-1098. doi: 10.1128/jvi.00877-10

Yan, Y. X., Du, Y. M., Wang, G. F., Deng, Y. X., Li, R., and Li, K. S. (2016). The novel H7N9 Influenza A virus NS1 induces p53-mediated apoptosis of A549 cells. Cell. Physiol. Biochem. 38, 1447-1458. doi: 10.1159/000443087

Ye, Q. Z., Krug, R. M., and Tao, Y. Z. J. (2006). The mechanism by which influenza A virus nucleoprotein forms oligomers and binds RNA. Nature 444, 1078-1082. doi: 10.1038/nature05379

Yi, C. Y., Zhao, Z. Z., Wang, S. Y., Sun, X., Zhang, D., Sun, X. M., et al. (2017). Influenza A virus PA antagonizes interferon-beta by interacting with interferon regulatory factor 3. Front. Immunol. 8:1051. doi: 10.3389/Fimmu.2017.01051

Yoshimura, A., Kuroda, K., Kawasaki, K., Yamashina, S., Maeda, T., and Ohnishi, S. (1982). Infectious cell entry mechanism of influenza virus. J. Virol. 43, 284-293.

Yount, J. S., Karssemeijer, R. A., and Hang, H. C. (2012). S-palmitoylation and ubiquitination differentially regulate interferon-induced transmembrane protein 3 (IFITM3)-mediated resistance to influenza virus. J. Biol. Chem. 287, 19631-19641. doi: 10.1074/jbc.M112.362095

Yount, J. S., Moltedo, B., Yang, Y. Y., Charron, G., Moran, T. M., Lopez, C. B., et al. (2010). Palmitoylome profiling reveals S-palmitoylation-dependent antiviral activity of IFITM3. Nat. Chem. Biol. 6, 610-614. doi: 10.1038/nchembio.405
Zhang, T. H., Ye, Z., Yang, X. H., Qin, Y. J., Hu, Y., Tong, X. M., et al. (2017). NEDDylation of PB2 reduces its stability and blocks the replication of Influenza A virus. Sci. Rep. 7:43691. doi: 10.1038/Srep43691

Zhao, C., Hsiang, T. Y., Kuo, R. L., and Krug, R. M. (2010). ISG15 conjugation system targets the viral NS1 protein in influenza A virus-infected cells. Proc. Natl. Acad. Sci. U.S.A. 107, 2253-2258. doi: 10.1073/pnas.09091 44107

Zheng, W., Cao, S., Chen, C., Li, J., Zhang, S., Jiang, J., et al. (2017). Threonine 80 phosphorylation of non-structural protein 1 regulates the replication of influenza A virus by reducing the binding affinity with RIG-I. Cell Microbiol. 19:e12643. doi: 10.1111/cmi.12643

Zheng, W. N., Li, J., Wang, S. S., Cao, S. S., Jiang, J. W., Chen, C., et al. (2015). Phosphorylation controls the nuclear-cytoplasmic shuttling of Influenza A virus nucleoprotein. J. Virol. 89, 5822-5834. doi: 10.1128/Jvi.00 015-5

Zurcher, T., Luo, G., and Palese, P. (1994). Mutations at palmitylation sites of the influenza virus hemagglutinin affect virus formation. J. Virol. 68, 5748-5754.

Conflict of Interest: The authors declare that the research was conducted in the absence of any commercial or financial relationships that could be construed as a potential conflict of interest.

Copyright (C) $2020 \mathrm{Hu}$, Zhang and Liu. This is an open-access article distributed under the terms of the Creative Commons Attribution License (CC BY). The use, distribution or reproduction in other forums is permitted, provided the original author(s) and the copyright owner(s) are credited and that the original publication in this journal is cited, in accordance with accepted academic practice. No use, distribution or reproduction is permitted which does not comply with these terms. 